\title{
LES LARVES DE GOERIDAE (TRICHOPTERA) DE LA FAUNE DE FRANCE. TAXONOMIE ET ECOLOGIE.
}

par S. Grenier ${ }^{1}$, H. Décamps ${ }^{2}$ et J. Giudicelli ${ }^{3}$.

\author{
SOMMAIRE
}

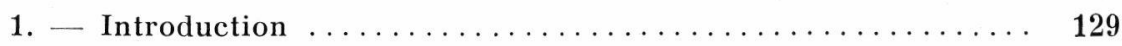

2. - Taxonomie larvaire ..................... 131

2.1. - Caractères généraux de la famille et nomenclature utilisée ....................... 131

2.2. — Description des espèces $\ldots \ldots \ldots \ldots \ldots \ldots \ldots \ldots \ldots 134$

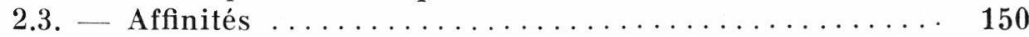

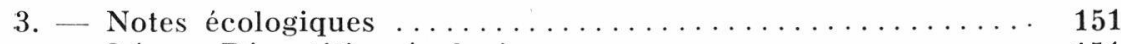

3.1. - Répartition écologique ................. 151

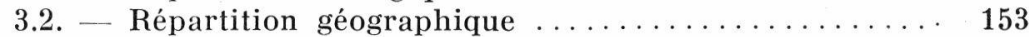

3.3. - Parasitisme ........................ 153

4. - Clé de détermination ....................... 154

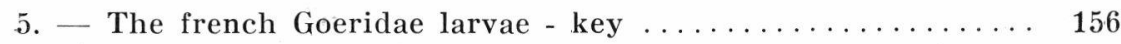

6. - Die französischen Goeriden-Larven - Bestimmungstabelle ... 158

\section{1. - INTRODUC'TION}

Les larves de la famille des Goeridae forment un élément constant de la faune pétrico!e des eaux courantes. Elles se nourrissent essentiellement de la couverture d'algues du substrat. Leur sténotopie remarquable en fait de bons indicateurs de zones : les espèces se succèdent régulièrement dans un même réseau hydrographique, depuis les sources jusqu'aux rivières de plaine [ILlies 1952, Dittmar 1955, Botosaneanu 1959, Adlmannseder 1965, Décamps 1968, GiUdicELli 1968].

1. Laboratoire de Biologie animale et Zoologie, Faculté des Sciences, 16, quai Claude-Bernard, 69-Lyon.

2. Laboratoire de Zoologie (E.R.A. d'Hydrobiologie), Faculté des Sciences, 118, route de Narbonne, 31 - Toulouse.

3. Laboratoire de Biologie animale (Ecologie), Faculté des Sciences, Traverse de la Barasse, 13 - Saint-Jérôme-Marseille. 
La détermination des larves de Goeridae est cependant rendue difficile par l'absence de descriptions suffisamment complètes et d'études comparatives. La plupart des espèces ne peuvent être distinguées avec certitude; les larves des deux espèces corses - Silo rufescens et Silonella aurata _. sont encore inconnues. Le but de cette note est d'établir une systématique des Goeridae, basée sur le cinquième stade larvaire, et utilisable par les hydrobiologistes. en Europe occidentale.

Nous présentons les neuf espèces de la faune française sur les onze connues en Europe occidentale. Ce sont :

Goera pilosa (Fabricius, 1775),

Lithax niger (Hagen, 1859),

Lithax obscurus (Hagen, 1859),

Silo graellsi E. Pictet, 1865,

Silo nigricornis (Pictet, 1834),

Silo pallipes (Fabricius, 1781),

Silo piceus (Brauer, 1857),

Silo rufescens (Rambur, 1842),

Silonella ${ }^{1}$ aurata (Hagen, 1864).

Les deux espèces absentes de France sont localisées, l'une en Espagne - Larcasia partita Navas, 1917 -, l'autre en Sardaigne - Silo mediterraneus (McL., 1884) - Cette dernière espèce est considérée comme douteuse [BotosanEanu, 1967].

Nous avons limité nos descriptions aux caractères discriminatifs. Certains caractères utilisés auparavant, sans grande signification taxonomique dans cette famille, n'ont pas été retenus : coloration, ligne latérale, branchies, crochets anaux... La capsule céphalique et les segments thoraciques ont été utilisés de préférence pour distinguer les espèces; ces parties chitineuses présentent l'avantage d'être retrouvées après la mue nymphale. Le genre Silonella, inconnu à l'état larvaire, fait l'objet d'une description plus complète.

Certains détails du relief tégumentaire étant d'une représentation difficile, nous complétons les dessins classiques par des clichés réalisés au microscope électronique à balayage.

Nous remercions nos collègues qui nous ont aidés à réaliser ce travail. I. Botosaneanu (Bucarest) et W. Döhi.eR (Klingenberg/Main) nous ont permis de connaître la larve de Lithax obscurus, M. Bounnaud et $\mathbf{H}$. TACHET (Lyon) ont mis à notre disposition du matériel de la région lyonnaise, B. Frochot (Dijon), J. Baudoin (Clermont-Ferrand) et M. Thibaurt (Biarritz) du matériel de Côte d'Or, du Massif Central et des Basses-Pyrénées.

1. Silonella Fischer $1966=$ Selis McL. 1876 [Frschen 1967]. 
J. M. Er.liotr (Ambleside) et W. Tobias (Frankfurt/Main) ont relu et corrigé nos clès de détermination en Anglais et en Allemand.

Les photographies au microscope électronique à balayage ont été réalisées grâce à l'amabilité de J. Chaline (Dijon) et R. Uzan (Lyon).

\section{2. - TAXONOMIE LARVAIRE}

\section{1. - Caractères généraux de la famille et nomenclature utilisée ${ }^{1}$.}

Les Goeridae ont des larves éruciformes, hypognathes, à tête rétractile dans le pronotum. Leur taille est moyenne $(7$ à $13 \mathrm{~mm}$ de longueur au dernier stade). Elles construisent un étui minéral.

L'étui est formé par un cylindre de sable possédant des expansions latérales plus ou moins développées (fig. 1 A-E). Ces expansions sont très réduites chez Lithax obscurus, avec des grains de sable identiques à ceux qui composent le cylindre (fig. $1 \mathrm{C}$ ); elles sont très développées chez Goera pilosa (fig. $1 \mathrm{~A}$ ). Les autres espèces constituent des cas intermédiaires: Lithax niger et toutes les espèces du genre Silo ont des étuis du même type (fig. 1 B). L'extrémité postérieure de l'étui est fermée par une membrane de soie percée d'un petit orifice circulaire. Sur cette membrane se trouvent parfois quelques grains de sable; c'est le cas dans le genre Lithax (fig. $1 \mathrm{E}$ ). La croissance s'effectue en cinq stades pendant lesquels la larve modifie fréquemment son étui [Nielsen 1942, Hansell 1968 ].

La capsule céphalique en vue dorsale, ou épicranium, présente trois éléments (fig. 2) : un frontoclypeus médian, deux pleures latéraux et symétriques. Les yeux, latéraux, sont réunis par une ligne interoculaire plus ou moins nette. Par rapport à cette ligne transverse, nous distinguons une zone antérieure et une zone postérieure. La zone postérieure peut être concave, aplatic ou convexe; la capsule céphalique a ainsi un profil anguleux ou arrondi (fig. 2 ).

Le pronotum, entièrement sc!érifié, possède deux pointes latéroantérieures. Il est plus ou moins convexe et présente parfois des zones en relief appelées protubérances. De plus, il peut être couvert de petits tubercules souvent sombres (fig. 2 ).

Le mésonotum, en grande partie sclérifié, comprend deux ou trois paires de sclérites médians, une paire de sclérites latéro-antérieurs et une paire de sclérites latéro-postéricurs. Les sclérites latéraux sont fusionnés chez Goera pilosa (fig. 4).

Le métanotum, en grande partie membraneux, ne présente que trois ou quatre paires de sclérites de petite taille.

1. Avant toute étude d'une larve, débarrasser tête et nota thoraciques des dépôts susceptibles de masquer les structures. 

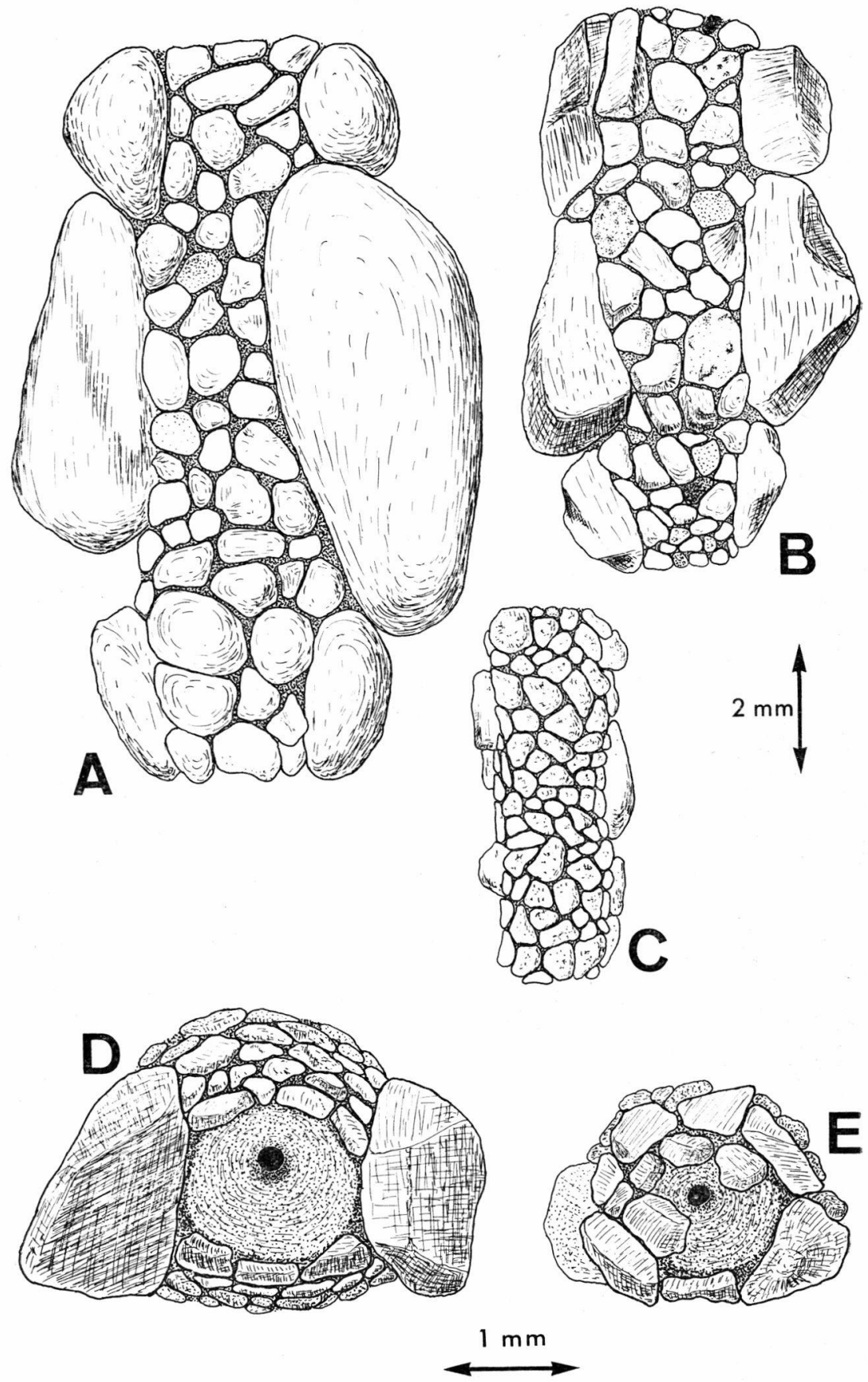

Fig. 1. - Etuis larvaires du dernier stade. A, B et C en vue dosrsale; D et E en vue postérieure. A : Goera pilosa; B et D : Silo piceus; C et E : Lithax obscurus. 

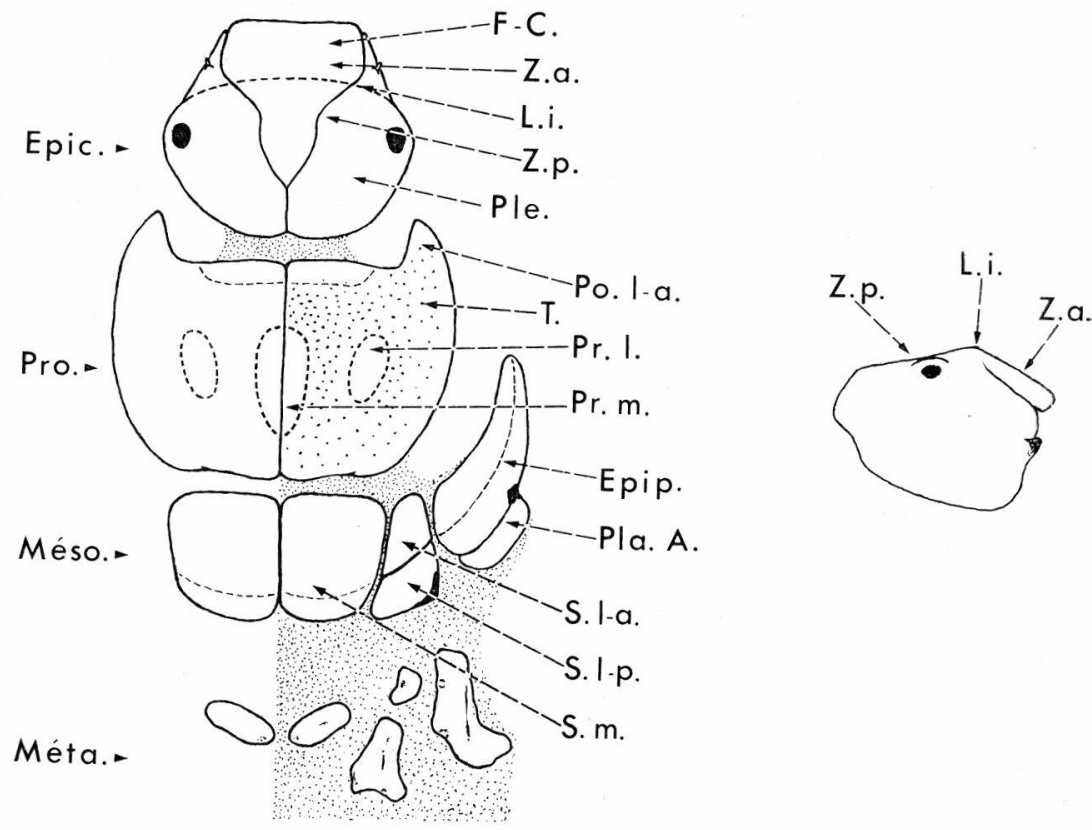

FIG. 2. - Aspect général d'une larve de Goeridae en vue dorsale et capsule céphalique vue de profil (Epic. : épicranium; Epip. : épipleure; F. C. : frontoclypeus; L. i. : ligne interoculaire; Méso. : mésonotum; Méta : métanotum; Pla. A. : plaque pleurale d'appui de la hanche; Ple. : pleure; Po. l-a. : pointe latéro-antérieure; Pr. 1. : protubérance latérale; $\mathrm{Pr} . \mathrm{m}$. : protubérance médiane; Pro. : pronotum; S. 1-a. : sclérite latéro-antérieur; S. 1-p. : sclérite latéro-postérieur; S. m. : sclérite médian; Z. a. : zone antérieure; Z. p. : zone postérieure).

Les trois paires de pattes sont sub-égales (fig. 3). Les tibias possèdent deux épines distales. Les hanches moyennes s'articulent sur une p'aque pleurale d'appui surmontée d'un prolongement, l'épipleure, qui borde latéralement le pronotum (fig. 2).

En résumé, les caractères qui permettent d'identifier les larves de Goeridae et de les distinguer, notamment de celles des Limnephilidae et Sericostomatidae, sont les suivants : forme de l'étui, relation entre tête et pronotum, aspect du pronotum, nombre de sclérites des méso- et métanotum et morphologie des pattes. D'autres caractères contribuent à préciser la diagnose : prégula transverse, postgula triangulaire et présence d'une corne prosternale. 


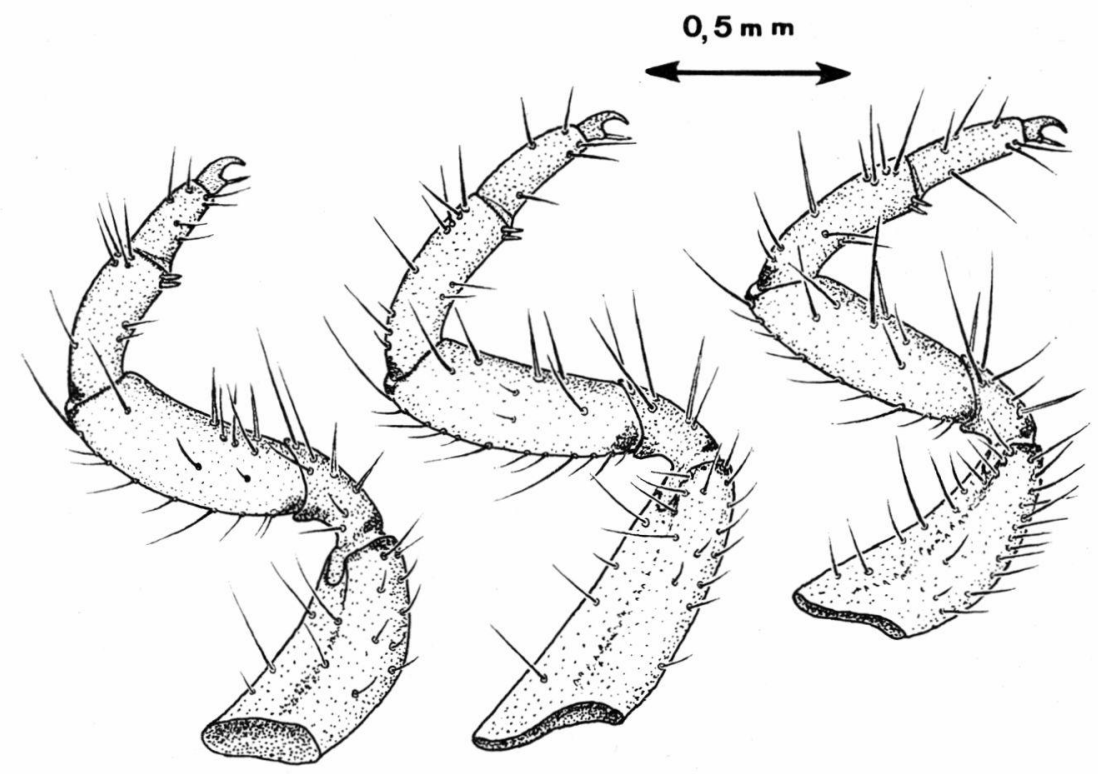

Fig. 3. - Pattes droites de Silo piceus en vue dorsale (l'avant est situé vers la gauche de la figure).

\section{2. - Description des espèces.}

Goera pilosa (fig. 4).

Matériel utilisé : nombreuses larves provenant de Bretagne, de Côte d’Or, du Jura, de la région lyonnaise et des Pyrénées.

Les principales descriptions antérieures de larves sont celles d'Ulmer [1909], Lestage [1921], Baker [1963], Lepneva [1966] et Hickin [1967].

Au cinquième stade, la taille maximale de la larve est de $13 \mathrm{~mm}$, la largeur du pronotum variant de 1,5 à $2 \mathrm{~mm}$. La longueur de l'étui est de 10-15 mm, sa largeur maximale, expansions comprises, atteint $10 \mathrm{~mm}$. Les expansions latérales sont très importantes, beaucoup plus que chez les espèces suivantes.

Parties chitinisées de couleur fondamentale claire. Capsule céphalique arrondie en vue dorsale, ne possédant pas de ligne interoculaire semblable à celle des espèces suivantes. Partie antérieure du frontoclypeus réduite. Pronotum plus large dans sa partie moyenne, présentant une protubérance médiane hérissée d'épines très courtes. Mésonotum avec deux paires de sclérites. Les sclérites latéraux, issus de la fusion de deux sclérites antérieur et postérieur, d'aspect triangulaire. Les sclérites médians garnis de soies courtes et épais- 

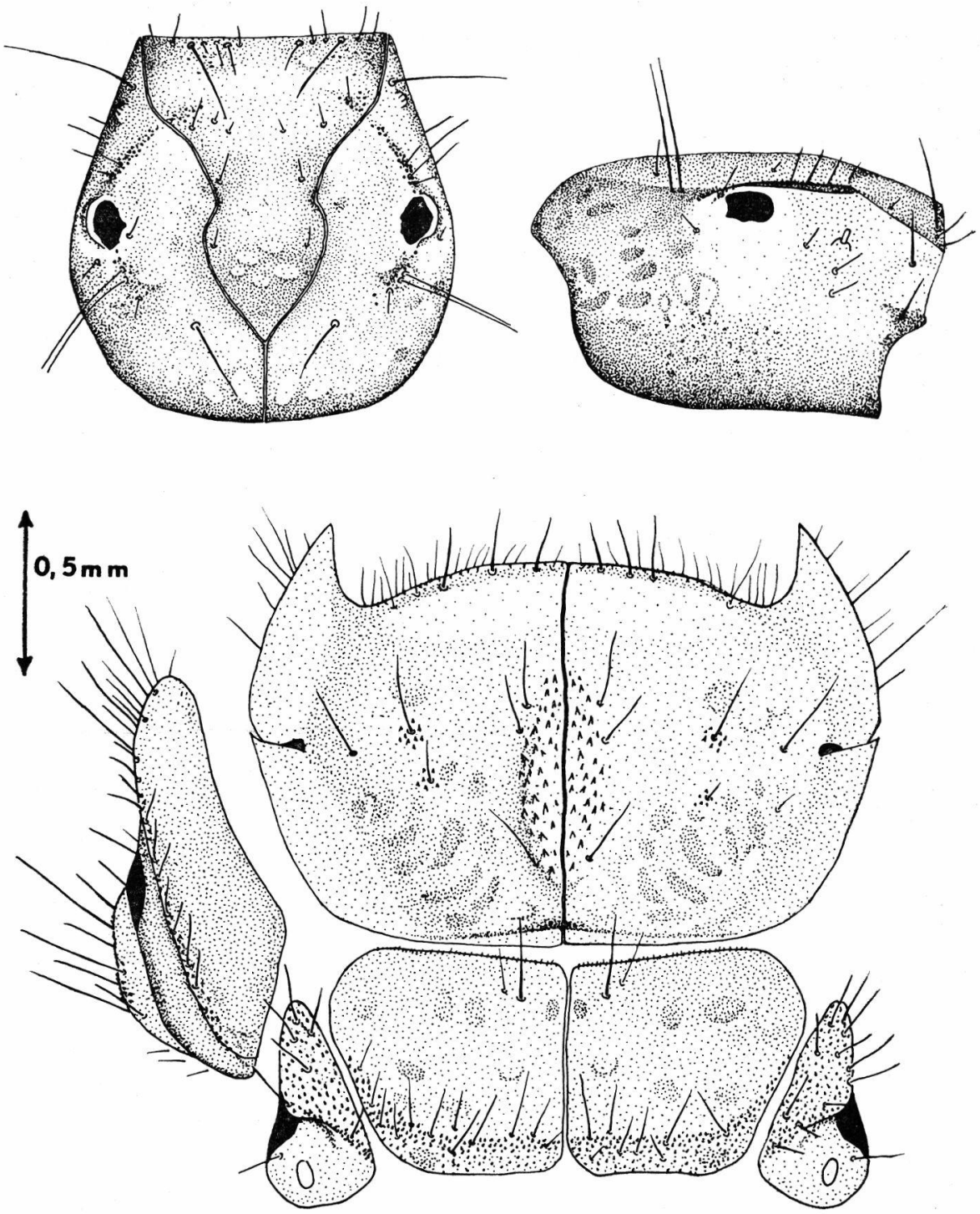

Fig. 4. - Larve de Goera pilosa: capsule céphalique en vue dorsale et de profil, pronotum, mésonotum. 
ses au bord antérieur. Métanotum avec trois paires de sclérites. La pilosité est dans l'ensemble beaucoup plus forte que chez les autres espèces; les fémurs ne présentent cependant que deux soies au bord antérieur.

Goera pilosa se distingue des espèces des autres genres, principalement par le nombre des sclérites méso- et métathoraciques. La forme arrondie de la capsule céphalique est un caractère commun à $G$. pilosa et Silonella aurata. La présence de deux soies au bord antérieur des fémurs se retrouve chez $G$. pilosa, $S$. aurata, et Lithax niger.
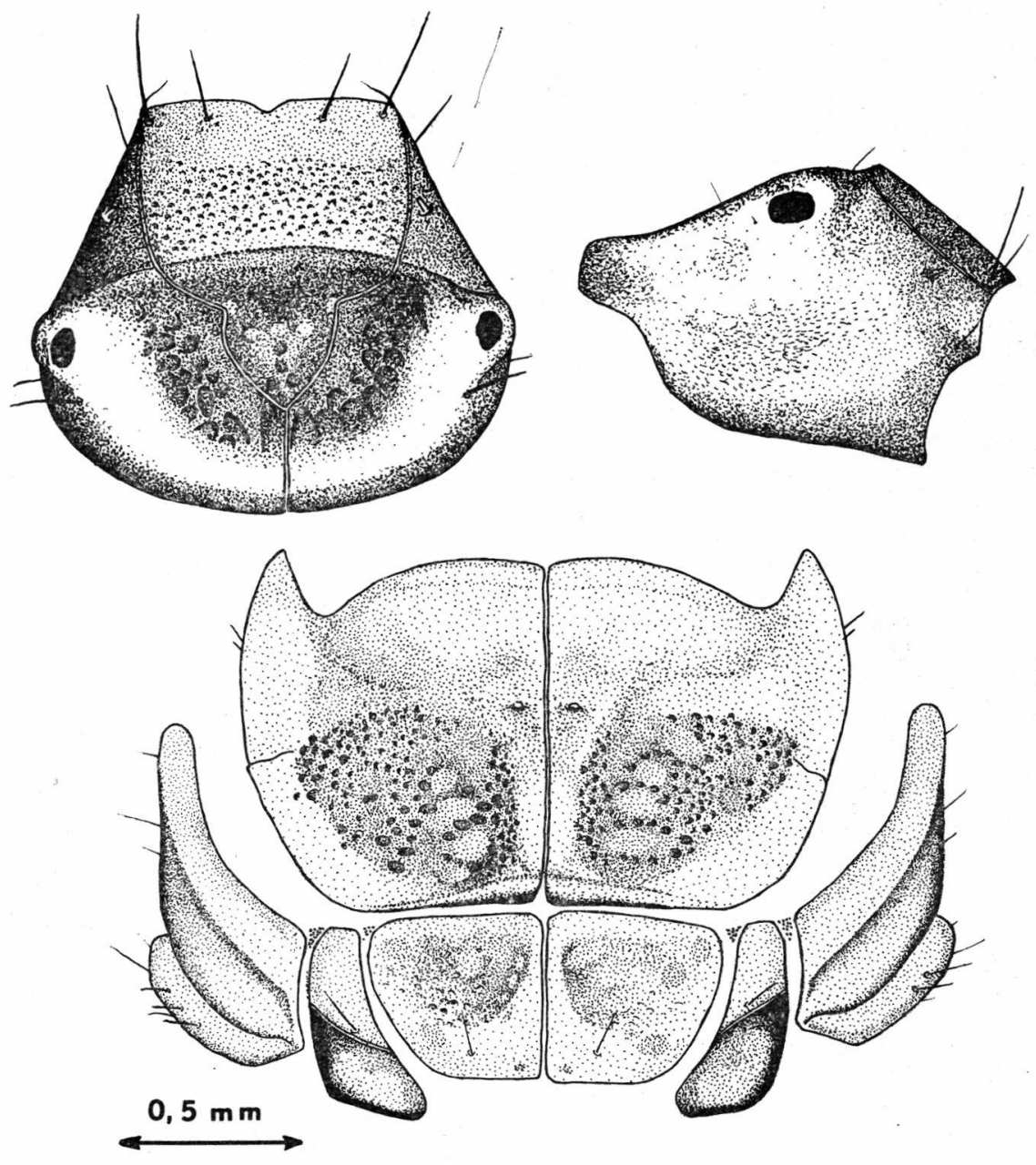

Fig. 5. - Larve de Lithax niger : capsule céphalique en vue dorsale et de profil, pronotum, mésonotum. 
Lithax niger (fig. 5, planches $1 \mathrm{C}$ et $2 \mathrm{C}$ ).

Matériel étudié : une quinzaine de larves de la région lyonnaise et grenobloise ainsi que du Massif Central.

Descriptions antérieures : KraWany [1932] et Botosaneanu [1952].

Taille maximale : $9 \mathrm{~mm}$. Largeur du pronotum : de 1,3 à $1,6 \mathrm{~mm}$. La longueur de l'étui varie de 8 à $10 \mathrm{~mm}$, sa largeur maximale est de $7 \mathrm{~mm}$. De petits cailloux sont présents sur la membrane postérieure de l'étui ( $c f$. fig. 1E).

Parties chitinisées de couleur fondamentale sombre. Capsule céphalique plus large que longue en vue dorsale. Ligne interoculaire très nette formant une crête aiguë en dents de scie et donnant un profil anguleux à la capsule céphalique. Zone postérieure de l'épicranium caractérisée par une très forte dépression. Cette dépression est tapissée de tubercules sombres. Elle est bordée vers l'arrière et sur les côtés par une zone plus claire, en relief. Pronotum élargi vers l'avant, pourvu de tubercules sombres vers l'arrière. Protubérance médiane s'évasant légèrement vers l'arrière et plus nettement vers l'avant. Mésonotum avec trois paires de sclérites. Bord antérieur des fémurs pourvu de deux soies.

\section{Lithax obscurus (fig. 6).}

Matériel utilisé : une vingtaine de larves provenant de Roumanie et d'Allemagne.

Les descriptions antérieures [ULmer 1909, Lestage 1921 et LePNEva 1966] reprennent celle d'Ulmer [1901].

Taille maximale : $7 \mathrm{~mm}$. Largeur du pronotum : 0,9 à $1,2 \mathrm{~mm}$. Longueur de l'étui : 6-7 mm pour une largeur maximale de $3 \mathrm{~mm}$, expansions comprises. Les expansions latérales sont très réduites et composées de cailloux identiques à ceux du cylindre. Comme chez Lithax niger, la membrane postérieure est garnie de petits cailloux (fig. $1 \mathrm{E}$ ).

Parties chitinisées de couleur fondamentale sombre. Epicranium et plaques pronotales garnies de petits tubercules plus ou moins sombres. Une ligne interoculaire peu marquée et une zone postérieure convexe confèrent un profil arrondi à la capsule céphalique. Pronotum sans protubérances, à pointes latéro-antérieures aiguës et à bords latéraux régulièrement arrondis. Mésonotum avec trois paires de sclérites. Epipleures non effilés antérieurement. Bords antérieurs des fémurs avec de nombreuses soies. 

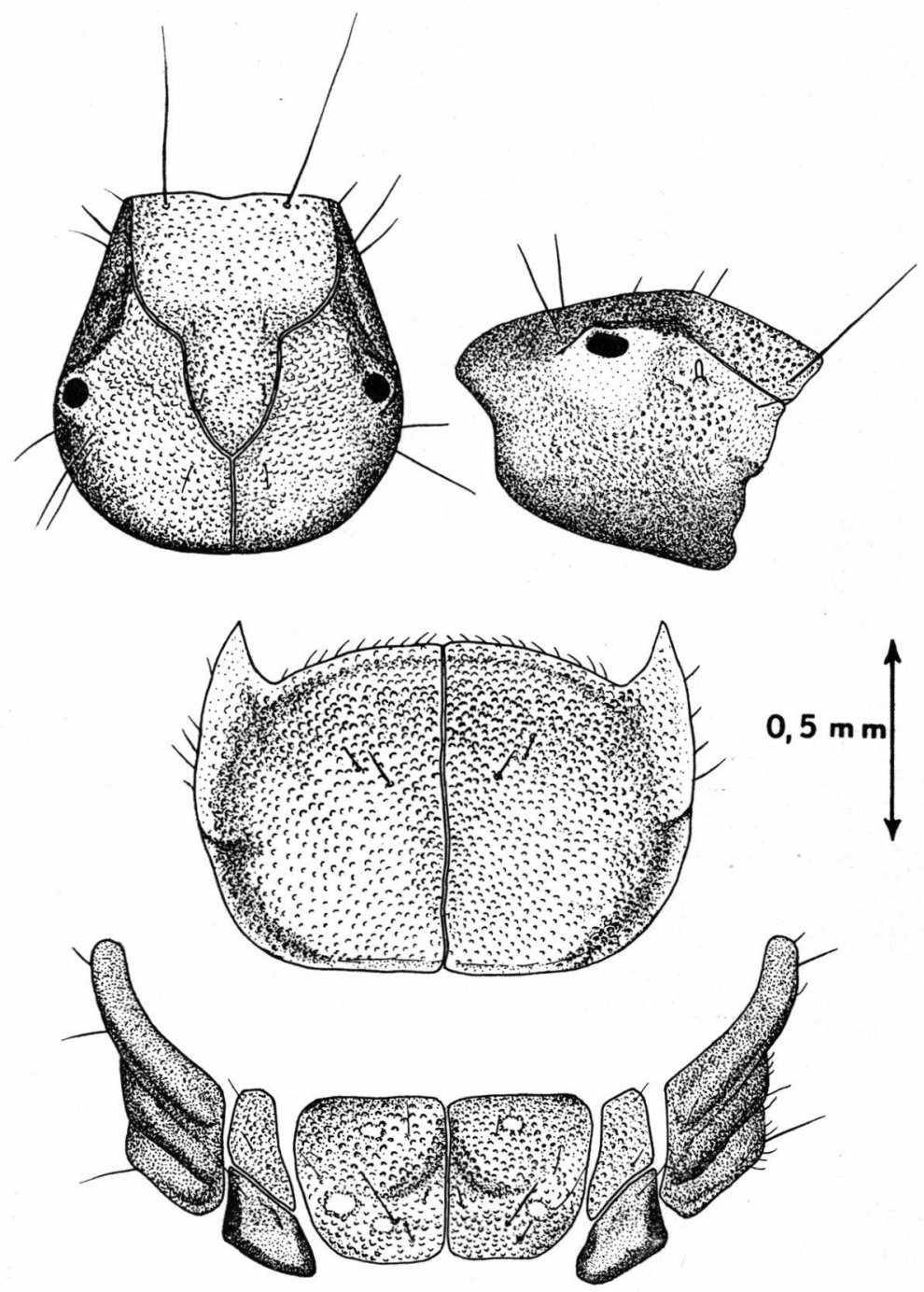

Fig. 6. - Larve de Lithax obscurus : capsule céphalique en vue dorsale et de profil, pronotum, mésonotum.

Silo graellsi (fig. 7, planches $1 \mathrm{~A}$ et $2 \mathrm{~A}$ ).

Matériel utilisé : nombreuses larves provenant des Pyrénées.

Seule description antérieure: Botosaneanu $[1956]$ s. n. Silo variipilosa Bots., considérée ensuite comme la sous-espèce $S$. Gracllsi variipilosa.

Taille maximale : $8 \mathrm{~mm}$. Largeur du pronotum : de 1,3 à 1,6 mm. Longueur de l'étui : 7-9 $\mathrm{mm}$, pour une largeur de $6 \mathrm{~mm}$, expansions 

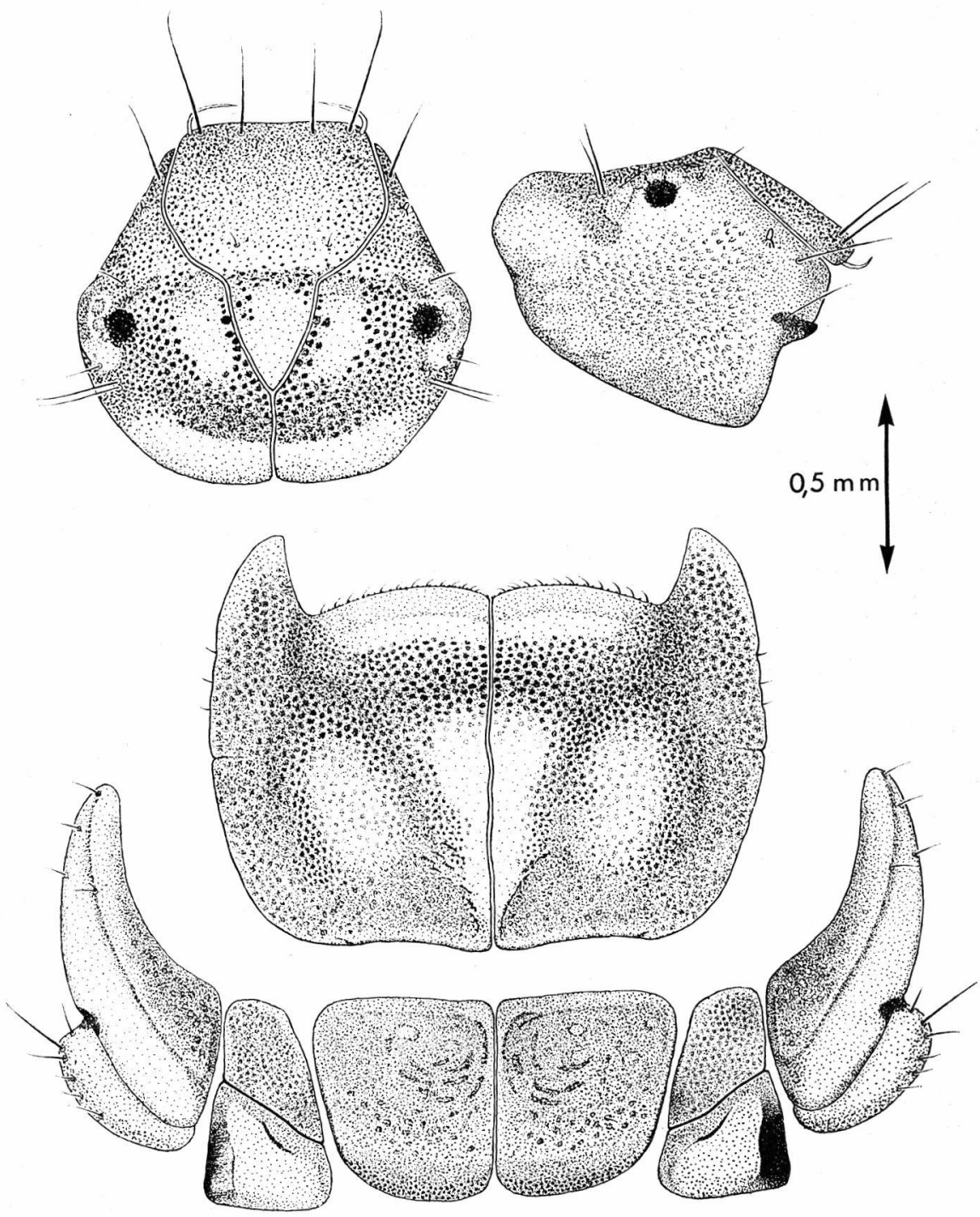

Fic. 7. - Larve de Silo graellsi : caspule céphalique en vue dorsale et de profil, pronotum, mésonotum. 

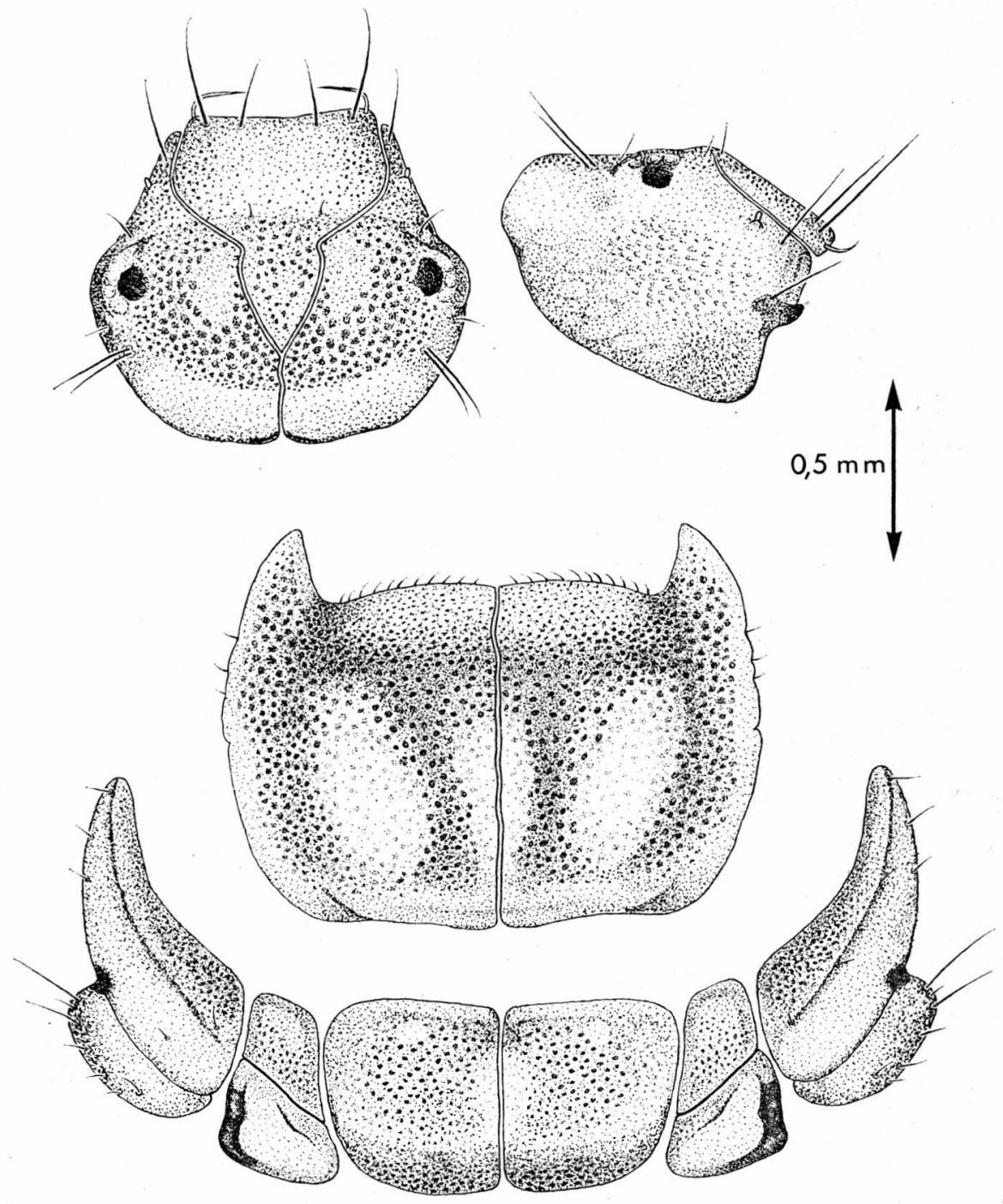

Fig. 8. - Larve de Silo pallipes : capsule céphalique en vue dorsale et de profil, pronotum, mésonotum. 
comprises. Expansions latérales moins importantes que chez Goera pilosa, beaucoup plus que chez Silonella aurata et Lithax obscurus.

Parties chitinisées de couleur fondamentale sombre. Capsule céphalique présentant une ligne interoculaire. Zone postérieure de l'épicranium légèrement déprimée, avec trois plages plus claires dépourvues de tubercules. Pronotum plus large dans sa partie moyenne, avec trois protubérances claires, la médiane d'aspect triangulaire, plus importante que les latérales. Bord antérieur du pronotum en relief, souvent de couleur plus claire, à limite antérieure échancrée en son milieu. La plus grande partie du pronotum est recouverte de tubercules foncés, sauf au niveau des protubérances où les tubercules sont plus clairs quand ils existent. Bord postérieur du pronotum et sclérites médians du mésonotum avec des dépressions punctiformes. Les fémurs présentent de nombreuses soies au bord antérieur.

Silo pallipes (fig. 8, planches $1 \mathrm{~B}$ et $2 \mathrm{~B}$ ).

Matériel utilisé : nombreuses larves provenant de la région lyonnaise.

Principales descriptions antérieures : NIELSEN [1942], BAKER [1963] et Lepneva [1966]. Sous le nom de $S$. pallipes, Ulmer [1909], Lestage : [1921] et Hickin [1967] ont confondu plusieurs espèces.

Taille maximale : $8 \mathrm{~mm}$. Largeur du pronotum : 1,2 à $1,5 \mathrm{~mm}$. Longueur de l'étui : 7-9 $\mathrm{mm}$, pour une largeur de $6 \mathrm{~mm}$, expansions comprises. Expansions latérales comme chez Silo graellsi.

Parties chitinisées de couleur fondamentale sombre. Capsule céphalique présentant une ligne interoculaire. Zone postérieure de l'épicranium aplatie, avec trois plages plus claires dépourvues de tubercules. Pronotum plus large dans sa partie moyenne, avec trois protubérances claires, la médiane plus petite ou, à la limite, de même importance que les latérales. Bord antérieur du pronotum très peu échancré en son milieu. La plus grande partie du pronotum est recouverte de tubercules sombres, sauf au niveau des protubérances où les tubercules sont plus clairs quand ils existent. Tous les reliefs sont moins accentués que chez Silo graellsi. Sclérites médians du mésonotum recouverts eux aussi de tubercules sombres. Fémurs présentant de nombreuses soies au bord antérieur.

Silo nigricornis (fig. 9, planche 2D).

Matériel utilisé : nombreuses larves provenant de la Côte d'Or, de la région lyonnaise et des Pyrénées.

Principales descriptions antérieures: NiElsen [1942], Baker [1963] et LEpNEva [1966]. D'autres descriptions sont insuffisantes 

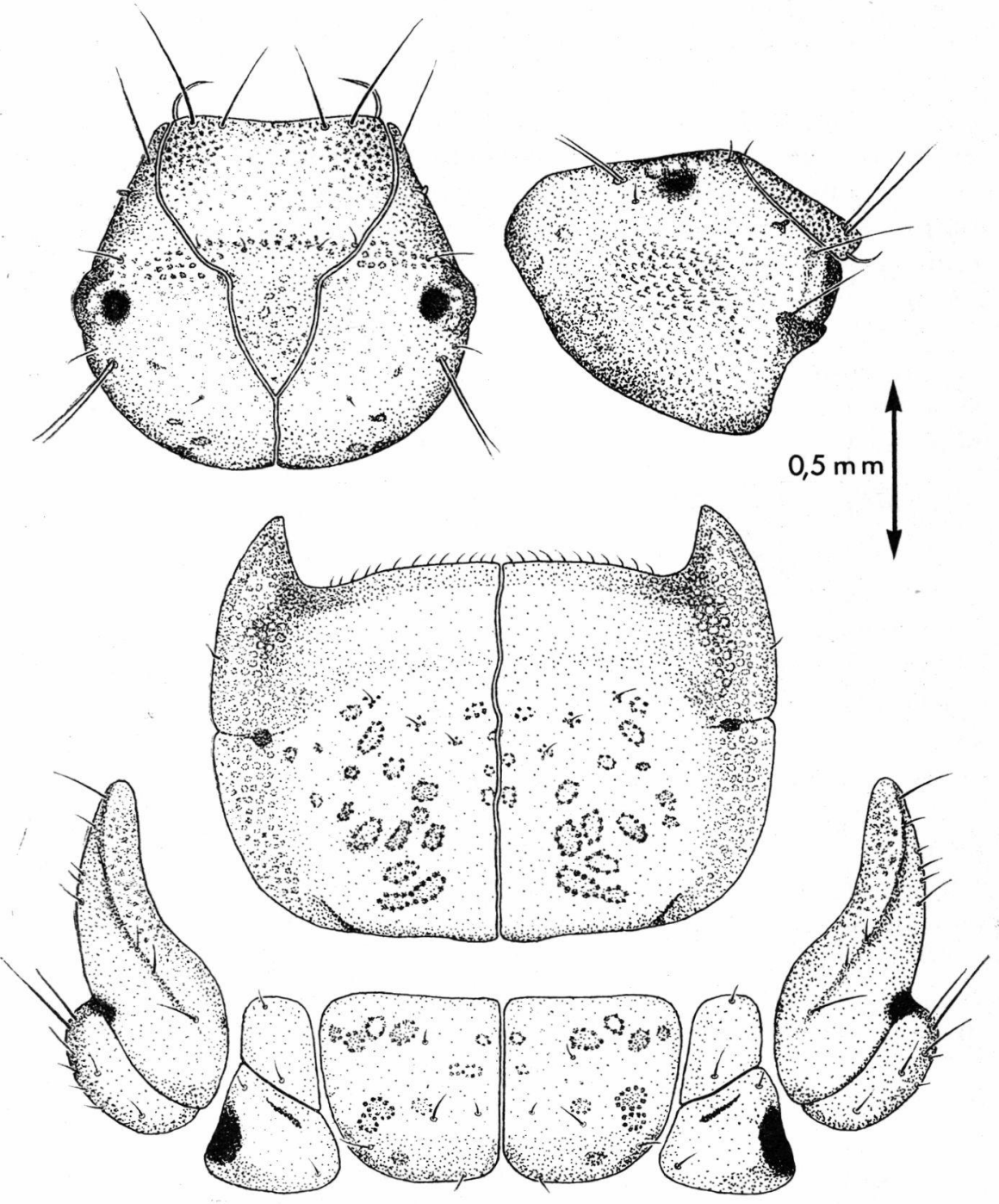

Fig. 9. - Larve de Silo nigricornis : capsule céphalique en vue dorsale et d€ profil, pronotum, mésonotum. 

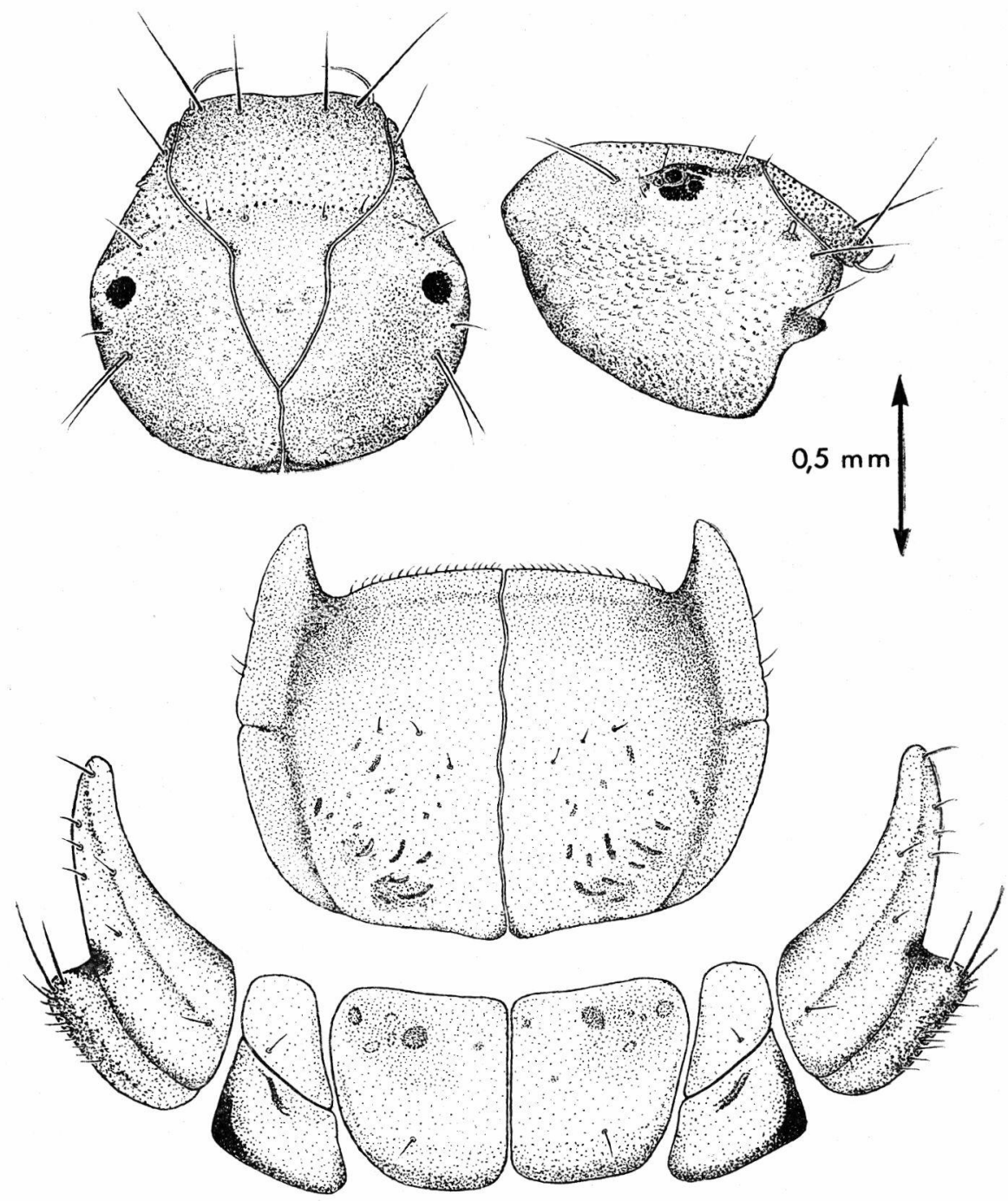

Fig. 10. - Larve de Silo piceus : capsule céphalique en vue dorsale et de profil, pronotum, mésonotum. 
[Ulmer 1909, Lestage 1921] ou se rapportent à plusieurs espèces [HICKIN 1967].

Taille maximale : $10 \mathrm{~mm}$. Largeur du pronotum : 1,4 à $1,8 \mathrm{~mm}$. Longueur de l'étui : 9-11 mm, pour une largeur de $8 \mathrm{~mm}$, expansions comprises. Expansions latérales comme chez Silo graellsi.

Parties chitinisées de couleur fondamentale claire. Capsule céphalique présentant une ligne interoculaire. Zone postérieure de l'épicranium aplatie. Pronotum plus large dans sa partie moyenne, sans protubérance, avec des tubercules clairs. Pronotum et sclérites médians du mésonotum avec plages d'insertions musculaires plus ou moins foncées, bordées d'une ligne de tubercules sombres. Fémurs présentant de nombreuses soies au bord antérieur.

Silo piceus (fig. 10, planches $1 \mathrm{~F}$ et $2 \mathrm{~F}$ ).

Matériel utilisé : nombreuses larves provenant de la région lyonnaise et des Pyrénées.

Les descriptions antérieures [Ulmer 1909 et Lestage 1921] sont imprécises et ne peuvent pas être utilisées.

Taille maximale : $9 \mathrm{~mm}$. Largeur du pronotum : 1,3 à $1,7 \mathrm{~mm}$. Longueur de l'étui : 8-10 mm, pour une largeur de $7 \mathrm{~mm}$, expansions comprises. Expansions latérales comme chez Silo graellsi.

Parties chitinisées de couleur très variable. En vue dorsale, capsule céphalique plus arrondie que celle des autres espèces du genre Silo. Ligne interoculaire présente. Zone postérieure de l'épicranium convexe, donnant un profil arrondi à la capsule céphalique. Pronotum p! lus large dans sa partie moyenne, sans protubérance ni tubercules. Pronotum et mésonotum le plus souvent de couleur claire avec des taches sombres au niveau des insertions musculaires, plus rarement uniformément sombre ou avec des plages plus claires mal délimitées. Fémurs présentant de nombreuses soies au bord antérieur (fig. 3).

Silo rufescens (fig. 11, planche 1D).

Matériel étudié : nombreuses larves de Corse.

Cette larve n'avait pas été décrite.

Taille maximale : 6,5 $\mathrm{mm}$. Largeur du pronotum : 1 à $1,4 \mathrm{~mm}$. La longueur de l'étui est de $6 \mathrm{~mm}$; sa largeur, expansions comprises, atteint $5,5 \mathrm{~mm}$.

Coloration générale brun ou brun-clair. Zone postérieure de la capsule céphalique déprimée. Ligne interoculaire en relief. Partic antérieure $d u$ frontoclypeus très élargie. Les tubercules tégumentaires ne sont pas très abondants; ils se répartissent sur la zone antérieure du frontoclypeus et sur le pourtour de la dépression céphalique (il n'y a aucun tubercule au centre de cette dépression). 

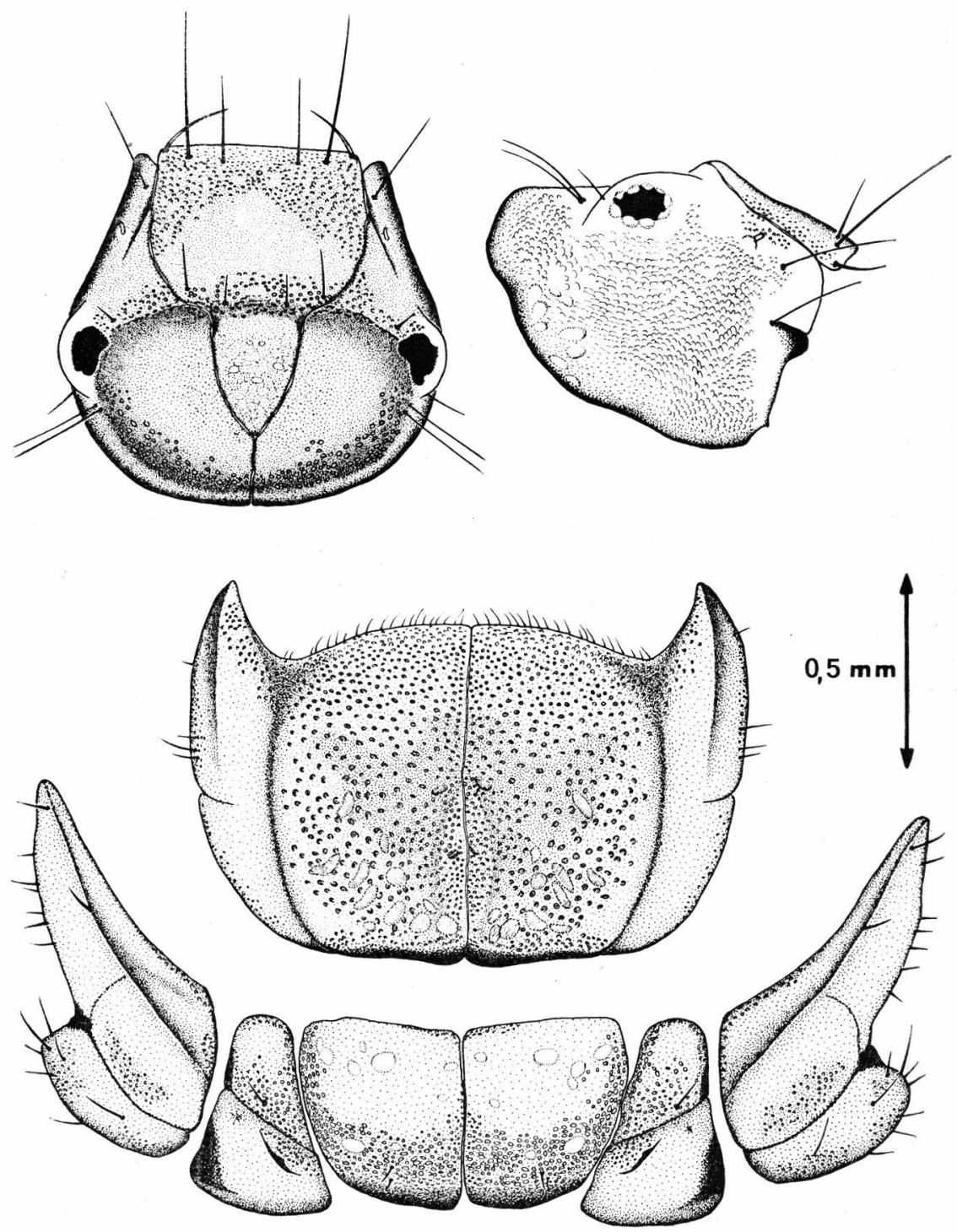

Fig. 11. - Larve de Silo rufescens : capsule céphalique en vue dorsale et de profil, pronotum, mésonotum. 
Pronotum plus large dans sa partie moyenne; il présente une protubérance médiane postérieure très peu saillante. Tubercules sombres à peu près uniformément répartis, très petits et plus denses au niveau de la protubérance médiane. Méso- et métanotum sans particularité. Pilosité identique à celle des autres larves de Silo.

Silo rufescens est remarquable par la dépression dorsale de la capsule céphalique. Par ce caractère, ele est proche de Silo graellsi, aucune autre espèce du genre ne présentant cette particularité. Cependant, Silo rufescens se distingue de Silo graellsi par la forme du pronotum : le bord antérieur est rectiligne chez rufescens et échancré chez graellsi, de plus, le pronotum de graellsi porte trois protubérances alors qu'il n'y en a qu'une chez rufescens. En outre, la ligne interoculaire forme une crête beaucoup plus aiguë chez. rufescens que chez graellsi.

Silonella aurata (fig. 12 à 14 , planches $1 \mathrm{E}$ et $2 \mathrm{E}$ ).

Matériel étudié : nombreuses larves de Corse.

Cette larve était inconnue jusqu'à présent.

Au 5ème stade la taille maximale de la larve est de $8 \mathrm{~mm}$; la largeur du pronotum varie de 1,0 à $1,3 \mathrm{~mm}$. La longueur de l'étui est de $7,5 \mathrm{~mm}$ pour une largeur de 2 à $3 \mathrm{~mm}$. Coloration générale brune, plus claire chez les larves prêtes à se nymphoser.

Capsule céphalique arrondie, un peu déprimée dorsalement. Ligne interoculaire bien marquée; au niveau du frontoclypeus, elle se traduit par un alignement caractéristique de 10 ou 12 gros tubercules arrondis, régulièrement espacés. La zone antérieure du frontoclypeus est moins large et la zone postérieure beaucoup moins étranglée en son milieu que chez les autres espèces de la famille. L'épicranium est légèrement déprimé en arrière de la ligne interoculaire; à l'intérieur de cette dépression la zone postérieure du frontoclypeus est saillante si bien que la dépression de l'épicranium a la forme d'un fer à cheval ouvert vers l'avant. Epicranium parsemé de nombreux petits tubercules sombres; ceux de la zone postérieure du frontoclypeus sont nettement plus gros.

Pronotum plus large en sa partic moyenne, à pointes latéro-antêrieures très aiguës. Une protubérance médiane en forme de triangle largement ouvert à l'avant et dont la pointe atteint presque le bord postérieur du pronotum. Mésonotum avec trois paires de sclérites, sans aucune particularité marquante. Métanotum avec quatre paires de sclérites.

La pilosité gẻnérale est peu abondante; ce caractère prend toute son importance au niveau des pattes (fig. 14) : les fémurs ne portent que deux soies à leur bord antérieur, une seule soie sur leurs faces externe et interne. Les crochets anaux sont tout à fait semblables à ceux des autres larves de Goeridae. 


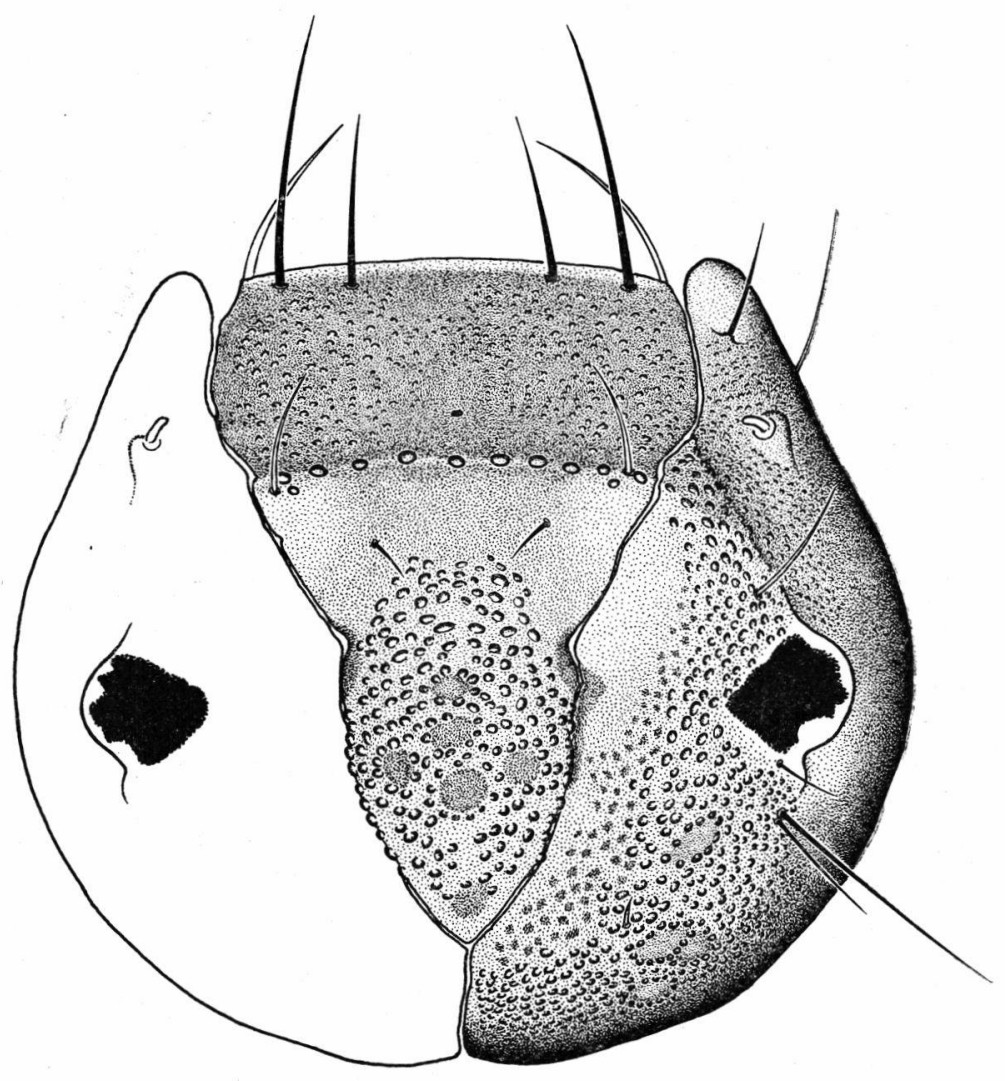

Fig. 12. - Larve de Silonella aurata: capsule céphalique en vue dorsale $(\times 90)$.

L'étui porte des surcharges latérales peu importantes; il paraît formé de 4 petits cylindres identiques superposés (fig. 14).

Silonella aurata se distingue des autres espèces de la famille par la forme triangulaire du frontoclypeus, par la présence d'un alignement transversal d'une dizaine de gros tubercules à la limite de la zone antérieure et de la zone postérieure du frontoclypeus, par la forme des pointes latéro-antérieures du pronotum, par la faible pilosité des pattes (caractère commun avec Lithax niger et Goera pilosa) et par l'étroitesse de l'étui (caractère commun avec Lithax obscurus). 


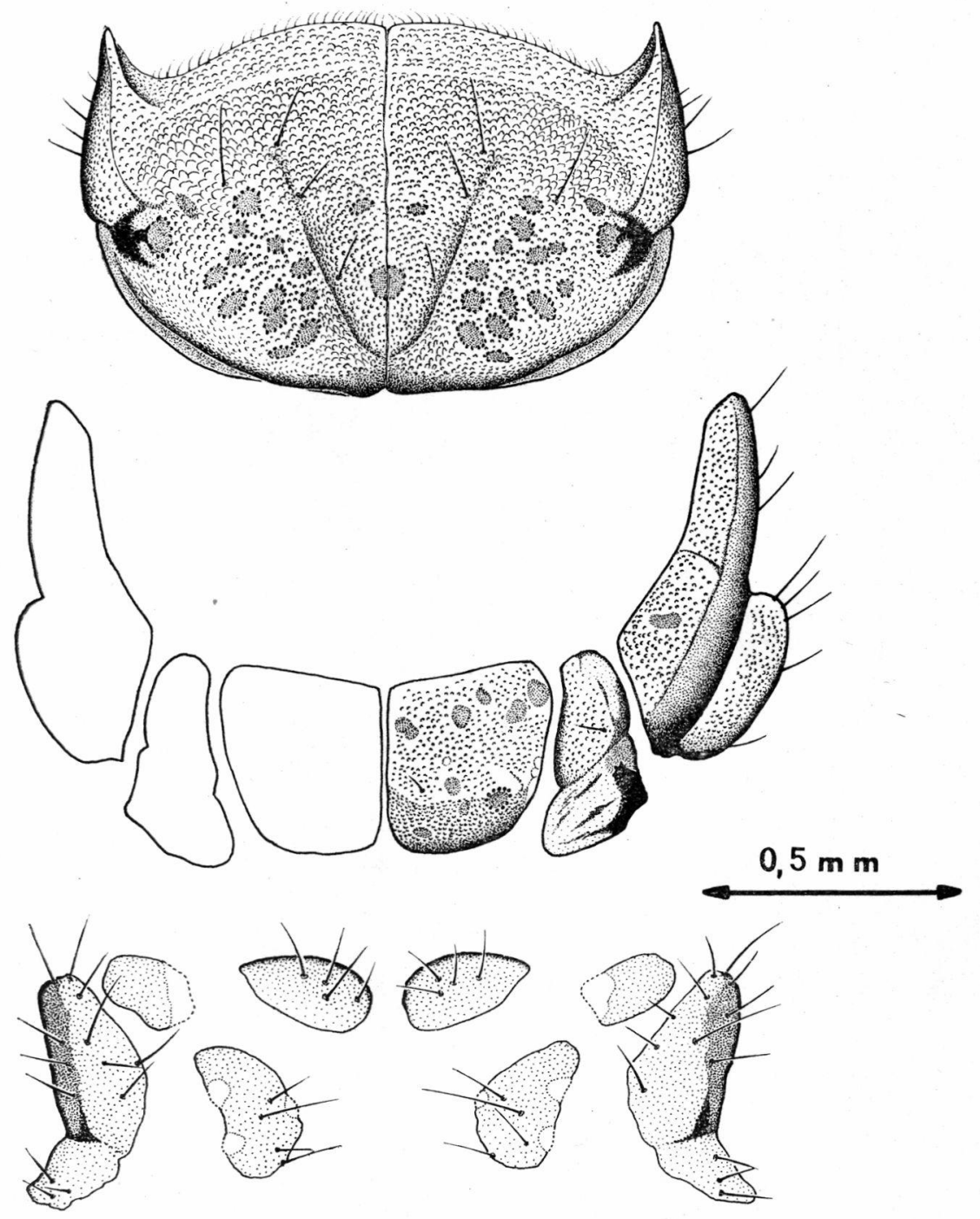

Fig. 13. - Larve de Silonella aurata : plaques thoraciques. 


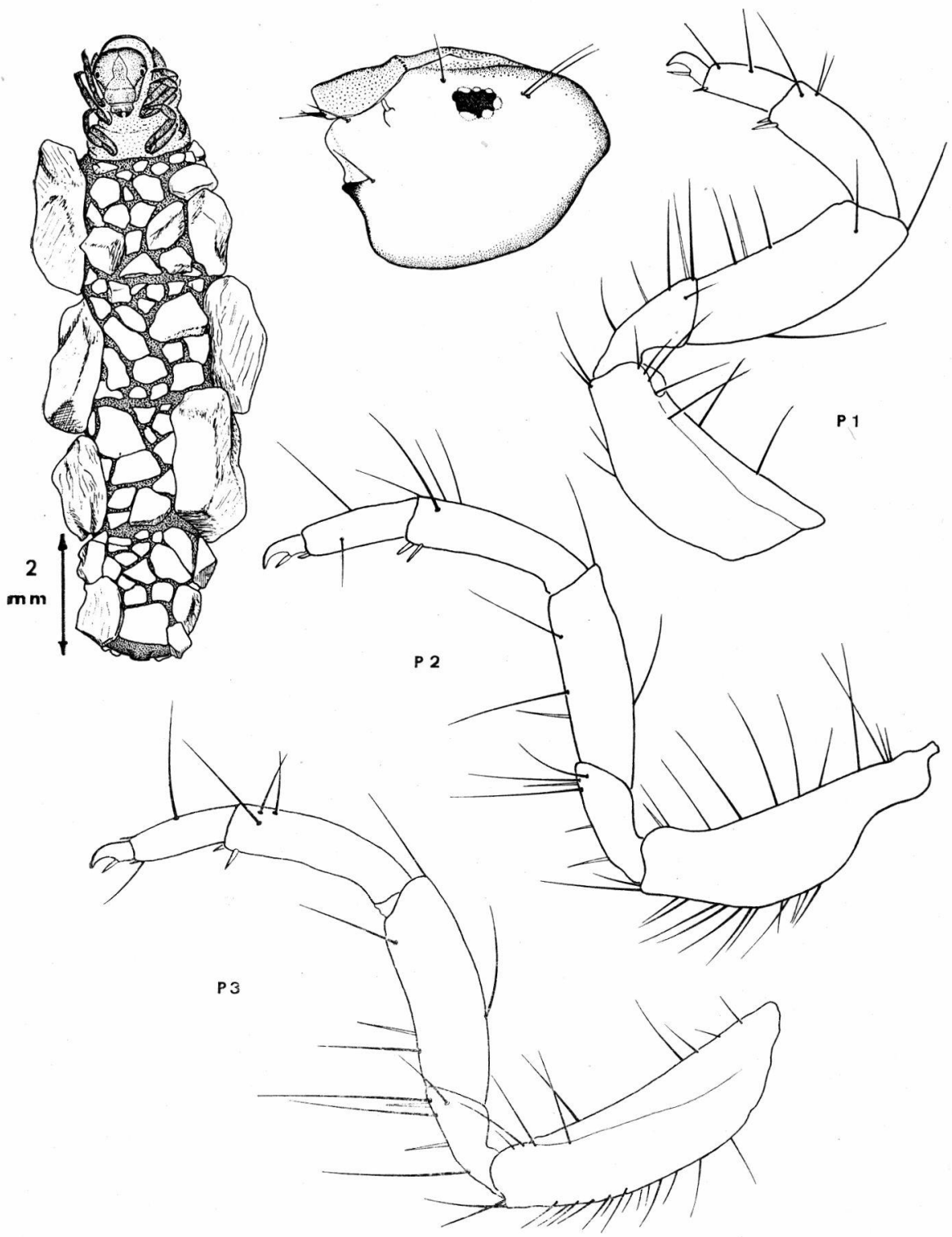

Fig. 14. - Larve de Silonella aurata : habitus, capsule céphalique vue de profil, pattes $(\times 40)$. 


\section{3. - Affinités.}

Goera pilosa se détache nettement des autres larves de la famille, tout d'abord par sa taille plus importante. Son caractère le plus original est représenté par la soudure des deux sclérites latéraux du mésonotum et la présence de trois paires de sclérites métathoraciques. Ces caractères sont d'ordre générique.

Tableau regroupant les différents Goeridae de la faune de France selon certains caractères larvaires communs.

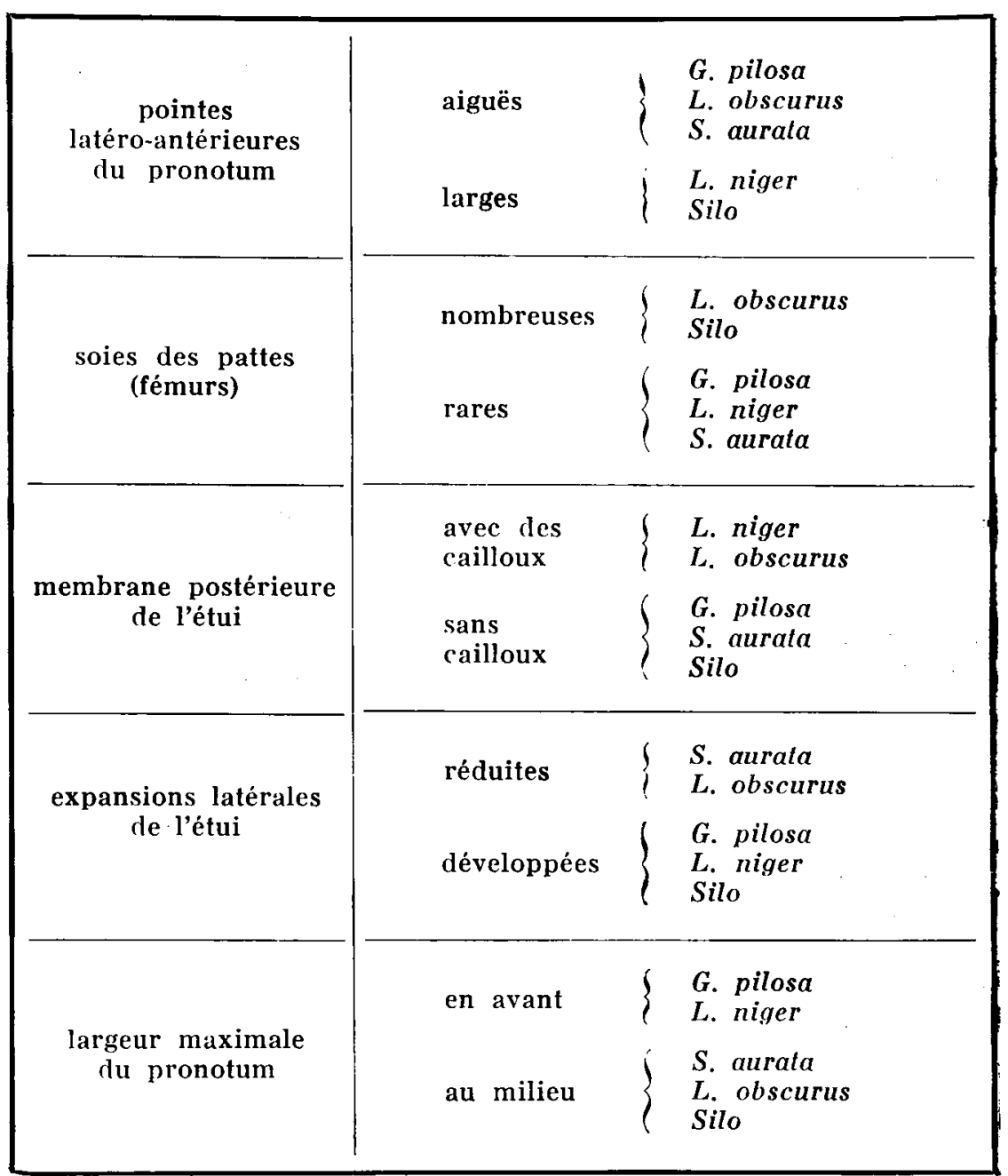



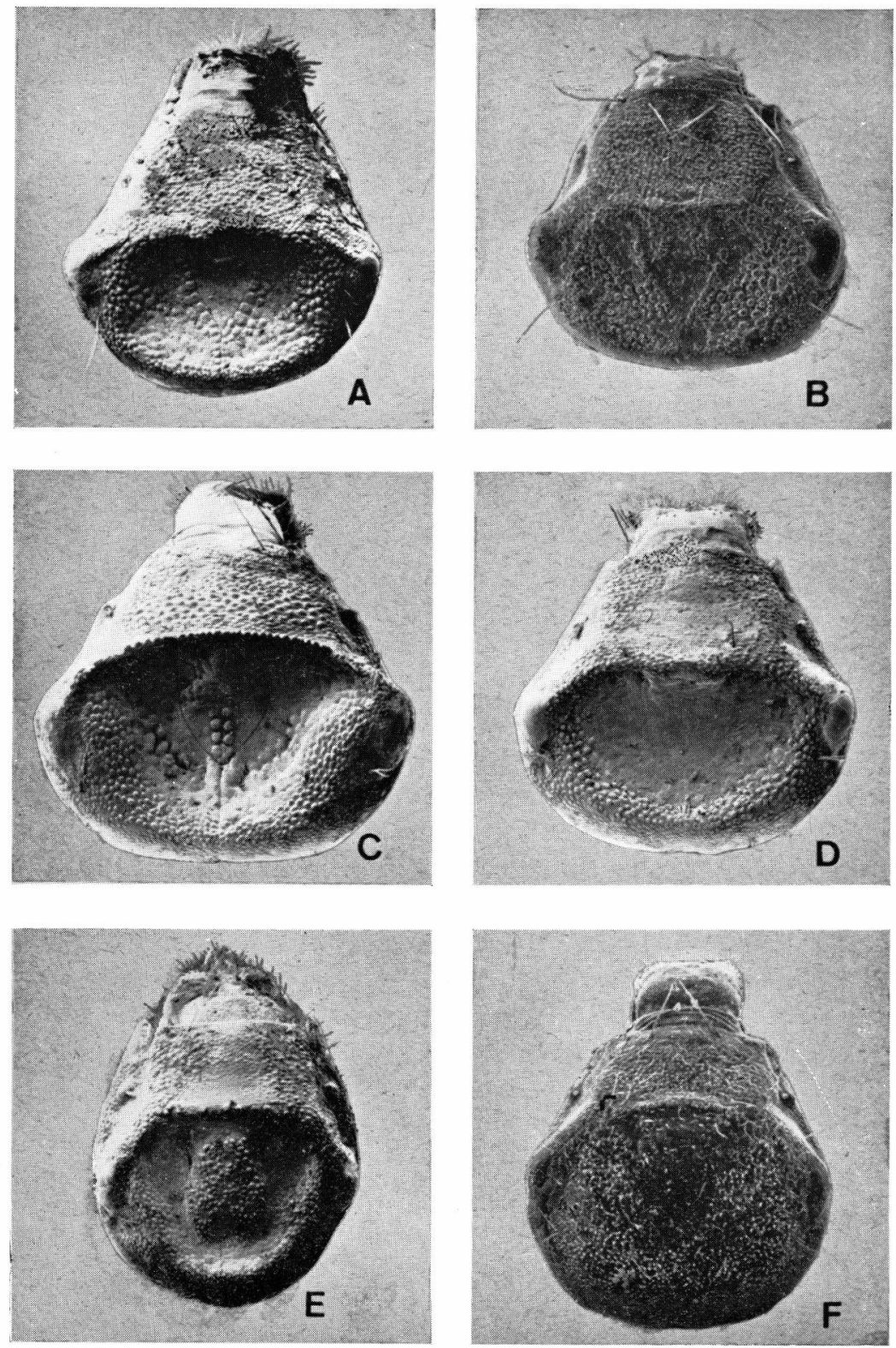

Planche 1. - Photographies au microscope électronique à balayage de la capsule céphalique en vue dorsale (épicranium). A : Silo graellsi ( $\times$ 42); B : Silo pallipes $(\times 37) ; \mathrm{C}:$ Lithax niger $(\times 40) ; \mathrm{D}$ : Silo rufescens $(\times 42) ; \mathbf{E}:$ Silonella aurata $(X 41) ; \mathrm{F}$ : Silo piceus $(\times 34)$. 

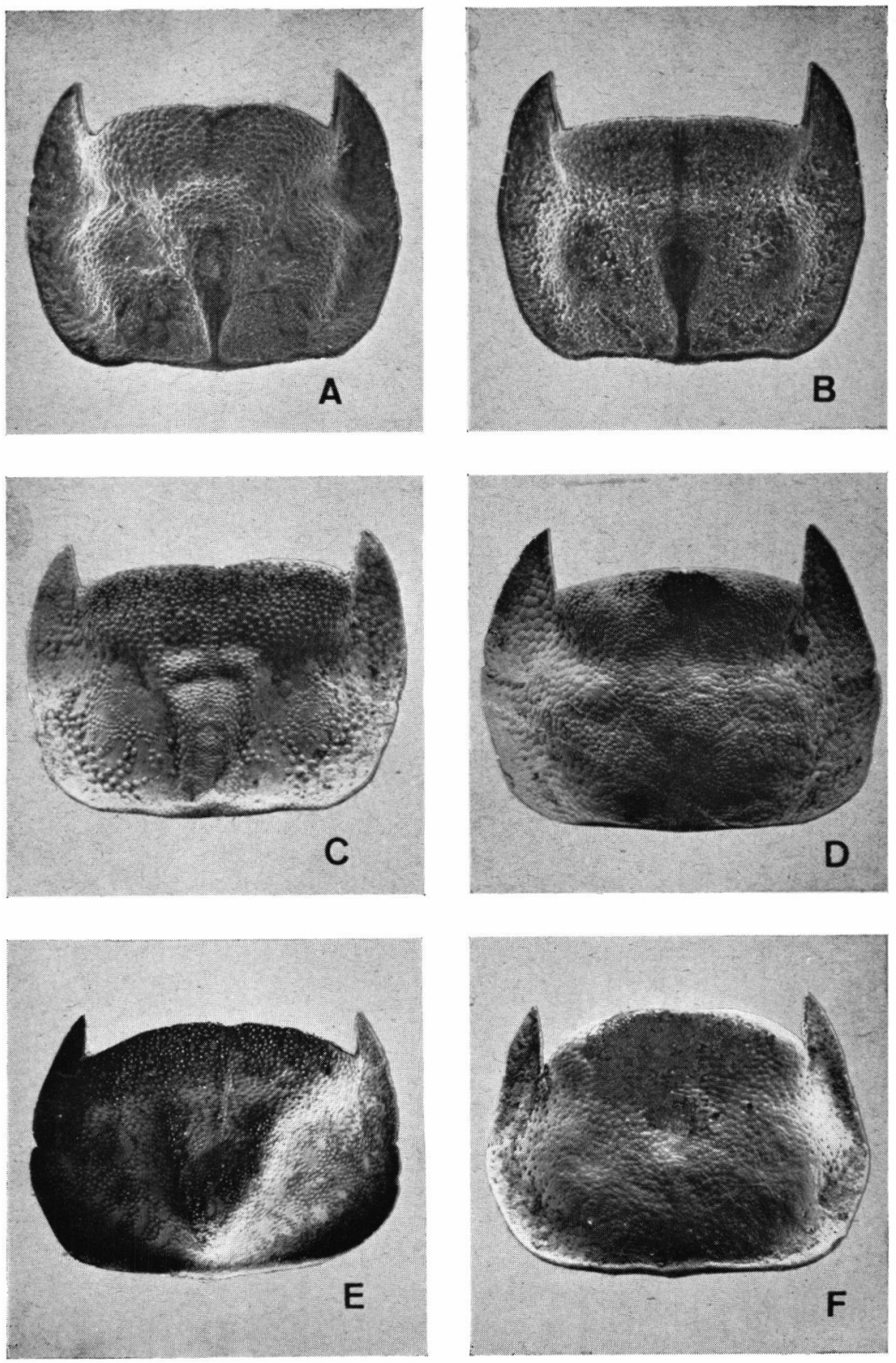

Planche 2. - Photographies au microscope électronique à balayage du pronotum. A : Silo graellsi $(\times 29)$; B : Silo pallipes $(\times 32)$; C : Lithax niger $(\times 33) ; \mathrm{D}$ : Silo nigricornis $(\times 30) ; \mathrm{E}$ : Silonella aurata $(\times 36)$; F : Silo piceus $(\times 33)$. 
Les trois autres genres de la famille ont en commun la présence de trois paires de sclérites au mésonotum et de quatre paires au métanotum. Deux genres, Silonella et Silo, paraissent bien individualisés par leurs caractères larvaires. Par contre, les deux espèces de Lithax sont tout à fait dissemblables. Elles diffèrent par de nombreux caractères : forme de la capsule céphalique, forme générale du pronotum, forme des pointes latérales du pronotum, pilosité des pattes. Elles n'ont en commun que la présence de petits cailloux sur la membrane postérieure de l'étui.

Dans le tableau ci-dessus, les espèces ont été groupées en fonction de certains caractères communs. Ces groupements sont très variables selon le caractère considéré. En particulier, la position des Lithax par rapport aux autres espèces de la famille est très imprécise. Les deux espèces du genre constituent, semble-t-il, deux rameaux divergents. Silonella aurata et Lithax niger forment un groupe à part, compte tenu de la faible pilosité de leurs pattes. De plus, Lithax obscurus et Silonella aurata sont remarquables par la forme générale de leur étui qui diffère de celle des autres espèces par des expansions latérales réduites.

La phylogénie de la famille ne saurait être retracée sans tenir compte des caractères imaginaux. Les caractères larvaires évoqués ici permettent cependant de concevoir, à partir d'une souche commune, l'existence de deux lignées : l'une avec Goera, l'autre avec Lithax + Silo + Silonella. Cette dernière lignée se ramifierait au niveau des Lithax, formant un premier rameau avec Lithax niger et Silonella. Les espèces du genre Silo se divisent en deux groupes suivant l'aspect de la capsule céphalique. Silo rufescens et $\boldsymbol{S}$. graellsi, avec une dépression dans la zone postérieure, forment un premier groupe. Les autres espèces, avec une capsule céphalique sans dépression, forment un second groupe.

\section{3. - NOTES ECOLOGIQUES}

\section{1. - Répartition écologique.}

Les larves de Goeridae sont des herbivores; elles se déplacent sur le substrat dont elles râclent la couverture biologique. Ce mode de vie leur est commun avec d'autres Trichoptères de la faune de France : Glossosomatinae, Thremma, et divers Limnephilidac. On les rencontre régulièrement dans les cours d'eau à fond pierreux. Leur distribution par rapport an courant est assez variable : Silo pallipes s'étale de 10 à $110 \mathrm{~cm} / \mathrm{s}$ dans une rivière du Cheschire [Scotr, 1958]; S. pallipes et $S$. nigricornis abondent de 40 à $70 \mathrm{~cm} / \mathrm{s}$ dans les ruisseaux de l'Himmerland [NIELSEN, 1942]; S. gr. nigri- 
cornis est peu rhéophile dans les sources de l'Ombrie (MoRETTI et Gianotri, 1962]; la densité de $S$. graellsi augmente jusqu’à $60 \mathrm{~cm} / \mathrm{s}$ dans les Pyrénées centrales [DÉcamps, 1968]. La distribution de l'espèce corse Silonella aurata reflète assez bien la variabilité des Goeridae par rapport au courant; son habitat le plus fréquent est formé par des zones calmes mais les larves peuvent être récoltées, agglutinées sous les pierres des rapides, jusqu’à $120 \mathrm{~cm} / \mathrm{s}$ [Giudicelli, 1968].
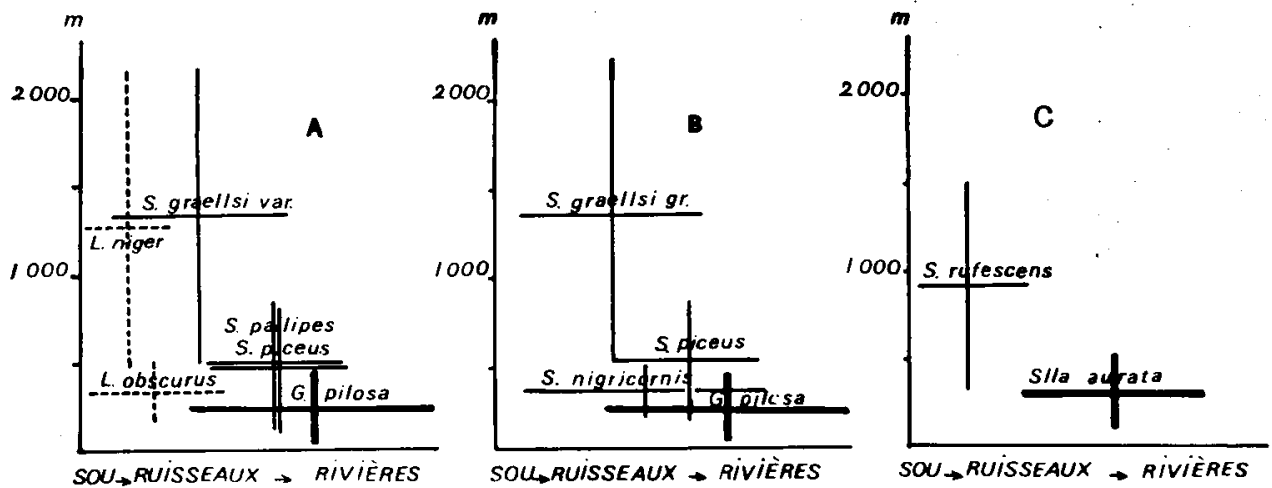

Répartition des Goerides dans les réseaux hydrographiques en fonction de l'altitude (ordonnée) et de l'importance du cours d'eau (abscisse). A : Roumanic'; B: Pyrénées; C : Corse.

La distribution écologique des Goeridae est bien connue dans trois régions montagneuses : en Roumanie [BotosanEaNU, 1959], dans les Pyrénées [DÉcamps, 1968] et en Corse [Givdicelli, 1968]. Les schémas ci-dessus représentent les positions approchées des espèces de la famille en fonction de l'altitude et de l'importance du cours d'eau (des sources jusqu'aux rivières). Les Lithax sont présents dans les sources et leurs émissaires à des niveaux différents, $L$. niger ayant une amplitude altitudinale plus importante que $L$. obscurus. Goera pilosa dans les Carpates et les Pyrénées et Silonella aurata en Corse peuplent les régions inférieures des réseaux hydrographiques. Les espèces du genre Silo s'échelonnent des sources jusqu'aux rivières. S. graellsi et $S$. rufescens fréquentent les régions des sources et montrent de grandes amplitudes altitudinales. En basse altitude, $S$. nigricornis s'étale des sources jusqu'aux rivières dans les Pyrénées. Silo pallipes et $S$. piceus vivent dans les grands ruisseaux et petites rivières de basse altitude. En Roumanie, Silo piceus a aussi été signalée dans les eaux stagnantes de moyenne montagne. 
Les mêmes données d'ensemble se retrouvent dans la région lyonnaise et le Dauphiné. Lithax niger se rencontre en altitude dans les Alpes grenobloises (déversoir de lac à $1600 \mathrm{~m}$ d'altitude) et dans le massif du Pilat, près de Saint-Etienne (ruisselet à $900 \mathrm{~m}$ d'altitude). Des adultes de Lithax obscurus ont été capturés près de Lyon, à $300 \mathrm{~m}$ d'altitude, aux abords d'un ruisseau de faible importance. En Auvergne, Lithax niger a été récoltée, surtout à l'état adulte, près de petites sources de 1600 à $1700 \mathrm{~m}$ sur les pentes du Plomb-de-Cantal.

La distribution des larves des espèces de Silo et Goera dans ces régions confirme la répartition altitudinale observée dans les Pyrénées et en Roumanie.

\section{2. - Répartition géographique.}

Quatre espèces ont une répartition relativement limitée. Citons d'abord les deux endémiques corses, Silo rufescens et Silonella aurata. Ce sont plus précisément des endémiques cyrno-sardes puisque McLachlan [1884] signale aussi leur présence en Sardaigne. Le genre Silonella existe encore en Afrique du Nord; Valluant [1954] a décrit une espèce d'Algérie, Silonella brevipalpis, connue seulement par l'imago, qui semble très proche de l'espèce insulaire. Le genre Silonella, présent uniquement en Afrique du Nord et en Corse-Sardaigne, fait partie de ces lignées sud-tyrrhéniennes que l'on trouve dans la faune Corse et qui constituent l'élément le plus ancien du peuplement de l'île [Grudicelli, 1968]. Une autre espèce, Silo graellsi a une aire sud-européenne : on la trouve dans la région pyrénéo-ibérique, dans les Balkans et les Carpates. Enfin, Lithax niger se cantonne dans le massif alpin, les Carpates et les massifs centraux hercyniens.

Les autres espèces françaises de Goeridae ont une répartition à peu près régulière qui s'étend à toute l'Europe.

\section{3. - Parasitisme.}

Les larves de Goeridae, avec leur étui homotypique par rapport au substrat, passent souvent inaperçues. Il n'en est pas de même pour certains étuis nymphaux présentant un long appendice rubané qui, depuis longtemps, a intrigué les hydrobiologistes.

Dès 1832, Cuntis décrivait Agriotypus armatus, mais ce n'est qu'en 1858, grâce à Von SIEBoLd, que le rapprochement était fait entre cet Hyménoptère Ichneumonoïde de la famille des Agriotypidae et le ruban ornant certains étuis de Goeridae.

Au printemps, les femelles d'Agriotypus descendent sous l'eau et recherchent les étuis nymphaux de Goeridae contenant une prénymphe ou une nymphe, afin d'y déposer leurs œufs. La larve du 
parasite se nourrit aux dépens de son hôte; ce dernier sera finalement entièrement consommé. La larve au dernier stade tisse, à l'intérieur de l'étui du Trichoptère, un cocon de soie prolongé à l'extérieur par un appendice rubané. Cet appendice aurait un rôle dans les échanges gazeux entre l'intérieur du cocon et l'eau environnante. La nymphose et la mue imaginale ont lieu en automne. L'insecte parfait passe l'hiver dans le cocon qui est rempli d'air. C'est au printemps suivant que l'imago sortira en découpant la paroi antérieure de son abri ${ }^{1}$.

Dans les biotopes favorables, 30 à $40 \%$ des prénymphes et nymphes peuvent être parasitées par cet entomophage.

La plupart des espèces de Goeridae de la faune de France (sauf trois) sont indiquées, dans la littérature comme parasitées par Agriotypus armatus. Goera pilosa, Silo nigricornis et Silo pallipes sont citées par de nombreux auteurs, parmi lesquels HENRiKsen [1922], Fisher [1932] et Messner [1965]. Silo piceus est citée par Müller [1889]. De même, Heymons $\lfloor 1909\rceil$ et Krawany [1942] indiquent respectivement Lithax obscurus et Lithax niger comme hôtes d'Agriotypus. Nous avons pu, en outre, observer le parasite chez Silo graellsi. Les espèces corses - Silo rufescens et Silonella aurata - n'ont encore jamais été trouvées parasitées. En France continentale, Agriotypus a été assez peu signalé. Il est cependant présent partout où se trouvent des Goeridae.

Agriotypus parasite deux familles de Trichoptères : les Goeridae et les Odontoceridae.

\section{4. - CLE DE DETERMINATION}

1. Mésonotum avec trois paires de sclérites (fig. 5 à 13). Métanotum avec quatre paires (fig. 13). Longueur maximale au dernier

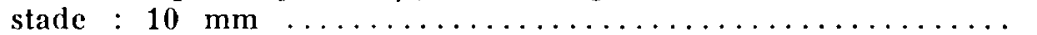

- Mésonotum avec deux paires de sclérites : sclérites latéraux antérieur et postérieur fusionnés, l'ensemble d'aspect triangulaire (fig. 4). Métanotum avec trois paires de sclérites. Longueur maximale au dernier stade $: 13 \mathrm{~mm}$......... Goera pilosa (Fbr.)

2. Capsule céphalique avec une très forte dépression dans la zone postérieure (fig. 5, planche $1 \mathrm{C}$ ). Cette dépression est bordée à l'avant par une crête aiguë en dents de scie (ligne interoculaire). Pronotum plus large dans sa partie antérieure, avec une protubérance médiane s'évasant légèrement vers l'arrière et largement vers l'avant (fig. 5, planche 2C). Etui possédant de petits cailloux sur la membrane postérieure ........... Lithax niger (Hag.)

- Capsule céphalique sans dépression ou avec une concavité bien moindre et non bordée antérieurement par une crête aiguë en dents

1. L'un d'entre-nous (S. Grenier) étudie actuellement la biologie d'Agriotypus. 
de scie. Pronotum plus large dans sa partie moyenne, sans protubérance, ou avec trois protubérances, ou encore avec une protubérance médiane non évasée aux deux extrémités. Etui le plus souvent sans petits cailloux sur la membrane postérieure (sauf

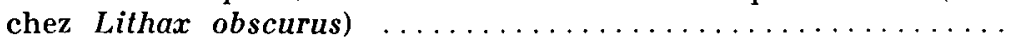

3. Pronotum avec trois protubérances plus ou moins claires (une mérliane, deux latérales). Tubercules foncés très apparents sur le pronotum. Capsule céphalique présentant un profil anguleux

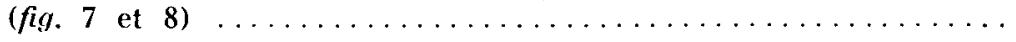

- Pronotum sans protubérance ou avec une protubérance, parfois peu accentuée et de même couleur que le reste du pronotum ou à peine plus pâle. Tubercules sombres du pronotum beaucoup plus petits, quand ils existent. Capsule céphalique à profil anguleux

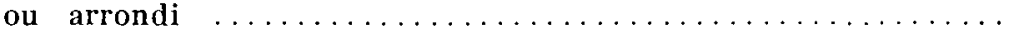

4. Pronotum avec la protubérance médiane plus importante que les latérales (fig. 7). Bord antérieur du pronotum en relief et largement échancré au niveau de la suture médiane (fig. 7 , planche $2 \mathrm{~A}$ ). Capsule céphalique avec une très légère dépression dans la zone postérieure (fig. 7, planche 1A) ........... Silo graellsi Pict.

- Pronotum avec trois protubérances de taille presque égale; la médiane plutôt plus petite (fig. 8). Bord antérieur du pronotum à peine échancré au niveau de la suture médiane (fig. 8 , planche 2B). Tous les reliefs sont en général moins accusés que chez Silo graellsi. Capsule céphalique aplatie dans la zone postérieure (fig. 8, planche 1B) ................. Silo pallipes (Fbr.)

5. Capsule céphalique sans dépression dans la zone postérieure (planche 1F). Pronotum sans protubérance (planche 2D et $\mathrm{F}$ ) ....

- Capsule céphalique avec une dépression simple (planche 1D) ou en forme de fer à cheval dans la zone postérieure (planche 1E). Pronotum avec une protubérance médiane plus ou moins accentuée

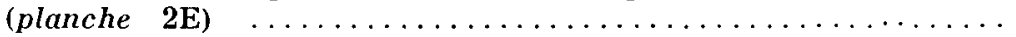

6. Epipleure, en vue dorsale, non effilé antérieurement (fig. 6). Pronotum à pointes latéro-antérieures aiguës et présentant de petits tubercules plus ou moins sombres sur toute sa surface (fig. 6). Capsule céphalique à profil arrondi (fig. 6). Etui à expansions latérales très faibles (fig. 1C), possédant de petits cailloux sur la membrane postérieure (fig. 1E) . . ...... Lithax obscurus (Hag.)

- Epipleure, en vue dorsale, effilé antérieurement (fig. 9 et 10). Pronotum à pointes latéro-antérieures assez larges (comme chez tous les Silo). Tubercules sombres du pronotum absents ou situés uniquement au niveau des insertions musculaires. Capsule céphalique à profil anguleux ou arrondi. Etui à fortes expansions latérales, sans cailloux sur la membrane postérieure (fig. 1B et D) ..

7. Capsule céphalique à profil anguleux (fig. 9). Pronotum en général clair avec des plages d'insertions musculaires bordées d'une ligne de tubercules sombres (fig. 9, planche 2D) .......... Silo nigricornis (Pict.)

-- Capsule céphalique à profil régulièrement arrondi (fig. 10). Pronotum de coloration très variable : a) souvent assez clair avec des taches sombres au niveau des insertions musculaires; b) plus 
rarement uniformément sombre ou, c) avec des plages plus claires mal délimitées. Tubercules sombres absents dans tous les cas (fig. 10, planche 2F) ................. Silo piceus (Brau.)

8. Protubérance du pronotum très peu accentuée, non triangulaire (fig. 11). Pronotum à pointes latéro-antérieures assez larges (comme chez les autres Silo). Capsule céphalique (fig. 11, planche 1D), avec une dépression dans la zone postérieure (un peu plus profonde que celle de Silo graellsi, mais bien moins que celle de Lithax niger) . . ................ Silo rufescens (Ramb.)

- Protubérance du pronotum accentuée, triangulaire, la pointe du triangle dirigée vers l'arrière (fig. 13, planche 2E). Pronotum à pointes latéro-antérieures très aiguës. Capsule céphalique avec une légère protubérance au niveau de la pointe postérieure du clypeus, entourée d'une dépression en forme de fer à cheval ouvert vers l'avant (fig. 12, planche 1E) ........ Silonella aurata (Hag.)

\section{5. — THE FRENCH GOERIDAE LARVAE - KEY}

1. Mesonotum with 3 pairs of sclerites (Fig. 5 to 13). Metanotum with 4 pairs of sclerites (Fig. 13). Maximal lenght when fully

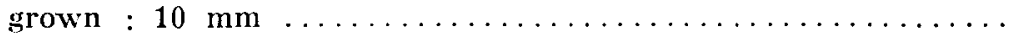

- Mesonotum with 2 pairs of sclerites: lateral triangular plate is the result of a fusion of the lateral anterior and lateral posterior plates (Fig. 4). Metatotum with 3 pairs of sclerites. Maximal length when fully grown : $13 \mathrm{~mm} \ldots \ldots \ldots \ldots \ldots$ Goera pilosa (Fbr.)

2. Head capsule with a very strong depression in the posterior part (Fig. 5, Plate 1C). This depression is borded on the anterior edge by a sharp ridge with saw-like teeth (this ridge forms the interocular line). Pronotum is widest in the anterior part and has a broad median ridge which widens towards the anterior and widens only slightly towards the posterior (Fig. 5, Plate 2C). Larval case with a posterior membrane covered with small stones ........ Lithax niger (Hag.)

- Head capsule without a depression, or with a less developed concavity and no saw-like teeth on the anterior ridge. Pronotum is widest in the middle part. When a broad median ridge is present, it does not widen towards the anterior and posterior. In most larval cases, posterior membrane without small stones (except in Lithax obscurus)

3. Pronotum with three raised areas which are more or less clear (one median and two lateral). Pronotum covered with small dark tubercles (chitinous processes) which are easily seen. Dorsal edge of head capsule angular in profile (Fig. 7 and 8) $\ldots \ldots$

- Pronotum without raised areas or with one median raised area, sometimes less pronounced and of the same colour as the rest of the pronotum or slightly paler. Tubercles on pronotum are very small when they are present. Dorsal edge of head capsule angular or curved in profile $\ldots \ldots \ldots \ldots \ldots \ldots \ldots \ldots$ 
4. Pronotum with the median raised area larger than the lateral raised areas (Fig. 7). Anterior edge of pronotum raised. Median suture of pronotum with large indentation at anterior end (Fig. 7, Plate 2A). Head capsule with a very slight concavity in the posterior part (Fig. 7, Plate 1A) .......... Silo graellsi Pict.

- Pronotum with three raised areas of almost equal size, with median area slightly smaller than lateral areas (Fig. 8). Median suture of pronotum with small indentations at anterior end (Fig. 8, Plate 2B). All the raised areas are generally less pronounced than those of Silo graellsi. Head capsule flat in the posterior part (Fig. 8, Plate 1B) ................. Silo pallipes (Fbr.)

5. Head capsule without a concavity in the posterior part (Plate 1F). Pronotum without any raised area (Plate $2 \mathrm{D}$ et $\mathrm{F}$ ) $\ldots \ldots \ldots \ldots$

- Head capsule with the posterior concavity either round (Plate 1D) or in the form of a horse shoe (Plate 1E). Pronotum with a median raised area more or less pronounced (Plate $2 \mathrm{E}) \ldots \ldots \ldots$

6. Epipleura, in dorsal view, not tapered anteriorly (Fig. 6). Pronotum has sharp anterior lateral projections, and is covered with small tubercles which are more or less dark (Fig. 6). Head capsule rounded in profile $(F i g .6)$. Lateral expansions poorly developed (Fig. 1C) and posterior membrane of case is covered with small stones (Fig. 1E) . . . . . . . . . . Lithax obscurus (Hag.)

- Epipleura, in dorsal view, tapered anteriorly (Fig. 9 and 10). Anterior lateral projections of pronotum are rather broad (as in all Silo species). Dark tubercles of pronotum absent or only occur in small patches. Hearl capsule in profle angular or curved. I-ateral expansions of case well developed and posterior membrane of case is not covered with stones (Fig. 1B and D) .......

7. Head capsule angular in profile (Fig. 9). Pronotum generally clear but with small rings of dark tubercles enclosing patches; these indicate the origin of muscles (Fig. 9, Plate 2D) ........

Silo nigricornis (Pict.)

- Head capsule in profile regularly curved, Color of pronotum very variable : it is, a) more frequently rather clear with dark patches, or b) more rarely uniformly dark, or c) more rarely with clear patches which are badly defined. In all cases dark tubercles absent (Fig. 10, Plate 2F) .................. Silo piceus (Brau.)

8. Raised area of pronotum not very obvious, not triangular (Fig. 11). Anterior lateral projections of pronotum rather broad (as in all Silo species). Head capsule (Fig. 11, Plate 1D) with a concavity in the posterior part (a little deeper than that of Silo graellsi, but not so deep as Lithax niger). Silo rufescens (Ramb.)

- Raised area of pronotum obvious, triangular, the acute point of the triangle projecting towards the posterior (Fig. 13, Plate 2E). Anterior lateral projections of pronotum very pointed. Head capsule with a slight raised area at the base of the posterior end of the clypeus, surrounded by a cavity in the form of a horse shoe open towards the anterior (Fig. 12, Plate 1E). Silonella aurata (Hag.) 


\section{6. - DIE FRANZöSISCHEN GOERIDEN-LARVEN - BESTIMMUNGSTABELLE}

1. Mesonotum mit 3 Paar (Fig. 5 - 13), Metanotum mit 4 Paar Schildchen (Fig. 13). Maximale Länge in letzten Stadium : $10 \mathrm{~mm}$

- Mesonotum mit 2 Paar Schildchen : die lateralen Schildchen (Vorder- und Hinterschildchen) verschmolzen, in der Gesamtansicht dreieckig (Fig. 4). Metanotum mit 3 Paar Schildchen. Maximale Länge in letzten Stadium : $13 \mathrm{~mm}$. Goera pilosa (Fbr.)

2. Kopfkapsel mit einer sehr starken Vertiefung in hinteren Teil (Fig. 5, Tafel 1C). Diese Vertiefung ist am Vorderrand von einem gezähnten, spitzen Kiel umsäumt (Zwischenaugen-Linie). Pronotum in seinen vorderen Teil breiter, mit einem medianen Höcker, der nach hinten nur wenig, nach vorn jedoch stark verbreitert ist (Fig. 5, Tafel 2C). Gehäuse mit kleinen Steinchen auf der hinteren Membran ............. Lithax niger (Hag.)

- Kopfkapsel ohne Vertiefung oder mit einer nur geringfüngigen Eindellung; vorn ohne gezähnten, spitzen Kiel. Pronotum in der Mitte breiter, ohne Höcker oder aber mit 3 Höckern oder auch mit einem Höcker in der Mitte, der jedoch nach vorn und nach hinten nicht verbreitert ist. Gehäuse meist ohne Steinchen auf der hinteren Membran (ausser bei Lithax obscurus) ............

3. Pronotum mit $3 \pm$ hellen Höckern (ein mittlerer, 2 seitliche), sowie sehr auffallenden dunklen Punkten auf dem Pronotum. Seitenansicht der Kopfkapsel eckig (Fig. 7 und 8) ........

- Pronotum ohne Höcker; manchmal mit einer schwach angedeuten Erhebung in der gleichen Farbe wie das übrige Pronotum oder ein wenig heller. Die dunklen Punkte des Pronotum sind, falls vorhanden, bedeutend kleiner. Seitenansicht der Kopfkapsel eckig

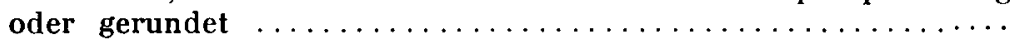

4. Mittlerer Höcker des Pronotum grösser als die seitlichen (Fig. 7). Vorderrand des Pronotum emporgewölbt und in der Mitte breit eingebuchtet (Fig. 7, Tafel 2A). Kopfkapsel mit sehr schwacher

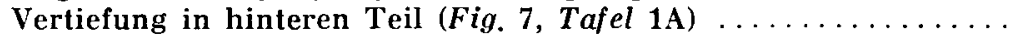
.............................. Silo graellsi Pict.

- Pronotum mit 3 beinahe gleichgrossen Höckern, der mittlere eventuell ein wenig kleiner als die übrigen (Fig. 8). Vorderrand des Pronotum in der Mitte kaum eingebuchtet (Fig. 8, Tafel 2B). Alle Erhebungen treten im allgemeinen weniger stark als bei Silo graellsi hervor. Kopfkapsel im hinteren Teil abgeflacht (Fig. 8, Tafel 1B) .................. Silo pallipes (Fbr.)

5. Kopfkapsel ohne Vertiefung in hinteren Teil (Tafel 1F). Pro-

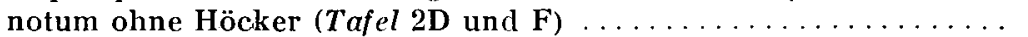

- Kopfkapsel mit einer einfachen (Tafel 1D) oder hufeisenförmigen Vertiefung im hinteren Teil (Tafel 1E). Pronotum mit einem \pm betont hervortretenden Höcker in der Mitte (Tafel 1E) .........

Epipleura, in Rückenansicht, vorn nicht ausgefasert (Fig. 6). Pronotum seitlich-vorn mit feinen Spizen und auf seiner ganzen 
Oberfläche mit kleinen, \pm dunklen Punkten versehen (Fig. 6). Seitenansicht der Kopfkapsel gerundet (Fig. 6). Gehäuse lateral sehr schwach verbreitert (Fig. 1C); hintere Membran mit kleinen Steinchen besetzt (Fig. 1E). ......... Lithax obscurus (Hag.)

- Epipleura, in Rückenansicht, in eine Spitze auslaufend (Fig. 9 und 10). Pronotum seitlich-vorn mit ziemlich breiten Spitzen (wie bei allen Silo-Arten). Dunkle Punkte des Pronotum fehlend oder ausschliesslich auf die Muskelansätze beschränkt. Seitenansicht der Kopfkapsel eckig oder gerundet. Gehäuse lateral stark verbreitert, ohne Steinchen auf der hinteren Membran (Fig. 1B

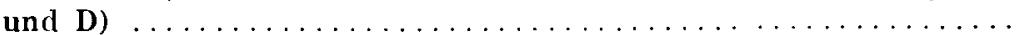

7. Kopfikapsel mit eckigem Seitenansicht (Fig. 9). Pronotum im allgemeinen hell, Muskelansätze von einer Reihe dunkler Punkte gesäumt (Fig. 9, Tafel 2D) ........... Silo nigricornis (Pict.)

- Kopfkapsel mit gleichmässig gerundetem Seitenansicht (Fig. 10). Färbung des Pronotum sehr veränderlich : a) häufig ziemlich hell, mit dunklen Flecken an dem Ansatzstellen der Muskeln; b) seltener einförmig dunkel oder c) mit unscharf umgrenzten helleren Flecken. In jedem Fall fehlen die dunklen Punkte (Fig. 10, Tafel 2F) .................... Silo piceus (Brau.)

8. Pronotum mit einem sehr schwachen, nicht dreieckigen Höcker (Fig. 11). Pronotum seitlich-vorn mit ziemlich breiten Spitzen (wie bei den anderen Silo-Arten). Kopfkapsel (Fig. 11, Tafel 1D) mit einer Vertiefung im hinteren Teil (etwas tiefer als die von Silo graellsi, jedoch nicht so tief wie die von Lithax niger) . . . . . Silo rufescens (Ramb.)

- Pronotum mit einem starken, dreieckigen Höcker; die Spitze des Dreiecks ist nach hinten gerichtet (Fig. 13, Tafel 2E). Pronotum seitlich-vorn mit sehr feinen Spitzen. Kopfkapsel mit unscheinbarem Höcker an der Hinterecke des Clypeus, umgeben von einer hufeisenförmigen, nach vorn geöffneten Rinne (Fig. 12, Tafel 1E) ...................... Silonella aurata (Hag.)

\section{TRAVAUX GITES}

AdimanNSEdEr (A.). 1965. - Faunistisch-ökologische Untersuchungen im Flussgebiete der Antiesen unter besonderer Berücksichtigung der Trichopteren. Jb. oberöst. MusVer., 110 : 386-421.

BAKer (W. V.). 1963. -- Some notes on the taxonomy of the last instar larvae of British species of the subfamily Goerinae (Trichoptera : Sericostomatidae). Proc, $R$. ent. Soc. Lond. (B), 32 : 171-174.

Botosaneanu (L.). 1952. - Contributii la studiul desvoltarii postembrionare si biologiei Trichopterelor : Rhyacophila laevis Pict. (Rhyacophilinae), Lype phaeopa Steph. (Psychomyinae), Ecclisopteryx guttulata Pict. (Ecclisopteryginae), Lithax niger Hag. (Goerinae). Buletin sti. Acad. Repub. pop rom., 4 (4) : 896-932.

Botosaneanu (L.). 1956. - Contribution à la connaissance des stades aquatiques des Trichoptères crénobiontes: Rhyacophila laevis Pict., Wormaldia triangulifera McLachl., Drusus romanicus Murg. et Bots., Silo variipilosa Bots. Beitr. Ent., 6 (5/6) : 590-624. 
Botosaneanu (L.). 1959. - Cercetari asupra Trichopterelor din Masivul Retezat si Muntii Banatului. Bibltca Biol. Animala, 1, 165 p. Bucarest.

Botosaneanu (L.). 1967. - Trichoptera, in Illies (J.) : Limnofauna Europaea, Stuttgart : 285-309.

Curtis (J.). 1832. - The genera of insects : Agriotypus armatus. Br. Entomology, 3 : 389.

Décamps (H.). 1968. - Vicariances écologiques chez les Trichoptères des Pyrénées. Annls Limnol. 4 (1) : 1-50.

Dittmar (H.). 1955. - Ein Sauerlandbach. Untersuchungen an einem Wiesen-Mittelgebirgsbach. Arch. Hydrobiol. 50 : 305-522.

Fischer (F. C. J.). 1967. - Trichopterorum Catalogus. Vol. VIII, Goeridae, Limnephilidae pars 1. Ned. Ent. Vereen. Amsterdam : vi +263 p.

Fisher (K.). 1932. - Agriotypus armatus (Walk.) (Hymenoptera) and its relations with its hosts. Proc. zool. Soc. Lond. : 451-461.

Grudicelli (J.). 1968. - Recherches sur le peuplement, l'écologie et la biogéographie d'un réseau hydrographique de la Corse centrale. (Thèse Sciences, Aix-Marseille, C.N.R.S. A. O. 2478 ), 437 p.

HANSELl (M. H.). 1968. - The house building behaviour of the caddis fly larva Silo pallipes Fabricius : I. The structure of the house and method of house extension. Anim. Behav., 16 : 558-561.

Henriksen (K. L.). 1922. - Notes upon some aquatic Hymenoptera. Annls Biol. lacustre, 11 : 19-37.

Heymons (R, und H.). 1909. - Hymenoptera in Brauer : Die Süsswasserfauna Deutschlands, Jena, 7 : 27-36.

Hickin (N. E.). 1967. - Caddis larvae, larvae of the British Trichoptera, $\mathrm{xI}+476$ p., London.

IllıEs (J.). 1952. - Die Mölle. Faunistisch-ökologische Untersuchung an einem Forellenbach im Lipper Bergland. Arch. Hydrobiol., 46 : 424-612.

Krawany (H.). 1932, - Trichopterenstudien in Gebiete der Lunzer Seen. Int. Revue Ges. Hydrobiol. Hydrogr. 26 : 388-396 et $27: 303-305$.

Krawaxy (H.). 1942. — Zur Entwicklung des Agriotypus armatus Walk. Int. Revue Ges. Hydrobiol. Hydrogr., 42 : 388-393.

Lepneva (S. G.). 1966. - La faune de l'U.R.S.S., Trichoptera, 2, 522 p. Moscou (en Russe).

Lestage (S. A.). 1921. - Trichoptera in Rousseau (E.) : les larves et nymphes aquatiques des insectes d'Europe. 967 p. Bruxelles.

McLACHLAN (R.). 1874-1884. - A monographic revision and synopsis of the Trichoptera of the european fauna, 523 p., pl. 1-51; Suppl. I et II : 1-103, pl. 52-59. First Additional Supll., 76 p., 7 pl. London.

Messner (B.). 1965. - Bemerkungen zur Biologie von Agriotypus armatus Walk. (Hymenoptera, Agriotypidae), Zool. Anz., 174 (4/5) : 354-362.

Moretti (G.) et Gianotri (F.S.). 1962. - Der Einfluss der Strömung auf die Verteilung der Trichoptera Agapetus gr. fuscipes Curt. und Silo gr. nigricornis Pict. Schweiz. Z. Hydrol., 24 : 467-484.

Müller (G. W.). 1889. -- Ueber Agriotypus armatus. Zool. Jb. Systematik, $4: 1132-1134$.

Nielsen (A.). 1942. - Ueber die Entwicklung und Biologie der Trichopteren mit besonderer Berücksichtigung der Quelltrichopteren Himmerlands. Arch. Hydrobiol., Suppl. $17: 255-631$. 
ScotT (D.). 1958. - Ecological Studies on the Trichoptera of the river Dean, Cheschire. Arch. Hydrobiol., 54 (3) : 340-392.

Siebold (von C.). 1858. - Ueber Agriotypus armatus und Trichostoma picicorne. Amtl. Ber. Vers. dt. Naturf. Carlsruhe, 34 : 211.

Ulmer (G.). 1901. - Beiträge zur Metamorphose der deutschen Trichopteren. Allg. Z. Ent. : 309-311.

Ulmer (G.). 1909. - Trichoptera, in Brauer : Die Süsswasserfauna Deutschlands, Jena, 5-6 : 1-326.

Vaillant (F.). 1954. - Three new species of Trichoptera from Algeria. Ann. Mag. nat. Hist., 12 (7) : 138-142. 